\title{
Article
}

\section{1,2,4-Oxadiazole-Based Bio-Isosteres of Benzamides: Synthesis, Biological Activity and Toxicity to Zebrafish Embryo}

\author{
Sen Yang, Chao-Li Ren, Tian-Yang Ma, Wen-Qian Zou, Li Dai, Xiao-Yu Tian, Xing-Hai Liu and Cheng-Xia Tan * \\ College of Chemical Engineering, Zhejiang University of Technology, Hangzhou 310014, China; \\ y17764528124@163.com (S.Y.); 18708589843@163.com (C.-L.R.); m15755187338@163.com (T.-Y.M.); \\ zwqxyes@163.com (W.-Q.Z.); 15157357729@163.com (L.D.); t895745887@163.com (X.-Y.T.); \\ xhliu@zjut.edu.cn (X.-H.L.) \\ * Correspondence: tanchengxia@zjut.edu.cn; Tel.: +86-571-8832-0238; Fax: +86-571-8832-0238
}

check for updates

Citation: Yang, S.; Ren, C.-L.; Ma, T.-Y.; Zou, W.-Q.; Dai, L.; Tian, X.-Y.; Liu, X.-H.; Tan, C.-X.

1,2,4-Oxadiazole-Based Bio-Isosteres of Benzamides: Synthesis, Biological Activity and Toxicity to Zebrafish Embryo. Int. J. Mol. Sci. 2021, 22, 2367. https://doi.org/10.3390/ ijms22052367

Academic Editor: Claudio Santi

Received: 17 January 2021

Accepted: 24 February 2021

Published: 27 February 2021

Publisher's Note: MDPI stays neutral with regard to jurisdictional claims in published maps and institutional affiliations.

Copyright: (c) 2021 by the authors. Licensee MDPI, Basel, Switzerland. This article is an open access article distributed under the terms and conditions of the Creative Commons Attribution (CC BY) license (https:/ / creativecommons.org/licenses/by/ $4.0 /)$.
Abstract: To discover new compounds with broad spectrum and high activity, we designed a series of novel benzamides containing 1,2,4-oxadiazole moiety by bioisosterism, and 28 benzamides derivatives with antifungal activity were synthesized. These compounds were evaluated against four fungi: Botrytis cinereal, FusaHum graminearum, Marssonina mali, and Thanatephorus cucumeris. The results indicated that most of the compounds displayed good fungicidal activities, especially against Botrytis cinereal. For example, 10a (84.4\%), 10d (83.6\%), 10e (83.3\%), 10f (83.1\%), 10i (83.3\%), and 101 $(83.6 \%)$ were better than pyraclostrobin $(81.4 \%)$ at $100 \mathrm{mg} / \mathrm{L}$. In addition, the acute toxicity of $\mathbf{1 0 f}$ to zebrafish embryo was $20.58 \mathrm{mg} / \mathrm{L}$, which was classified as a low-toxicity compound.

Keywords: benzamides; biological activity; 1,2,4-oxadiazole; synthesis; toxicity

\section{Introduction}

Chemical pesticides play a vital role in solving food problems. However, as people's environmental awareness gradually deepens, high-efficiency, low-toxicity, and environmentally friendly pesticides have become an inevitable trend in the creation of new pesticides [1-3]. There is no doubt that heterocyclic structures are important feature in synthetic pesticides for their high-efficiency, various biological activities, and diversity of possible substituents [4-25]. 1,2,4-Oxadiazole heterocycle, as an important kind of the five-membered oxygen-nitrogen heterocycle, has exhibited a wide range of biological activities in the field of pesticides, such as insecticidal [26-28], antifungal [29,30], and herbicidal activities [31]. It has often been introduced as a synergist into the structure of pesticides in order to improve the biological activities of the compounds. In addition, the 1,2,4-oxadiazole heterocycle, as a bio-isostere of the amide bond, has better hydrolytic and metabolic properties.

Diamide insecticides have attracted a lot of attention due to their novel mechanism of action, high efficiency, and low toxicity [32-42]. Following Bayer's report on the first diamide insecticide flubendiamide, the use of chlorantraniliprole, cyantraniliprole, cyclaniliprole, (Figure 1) and other products was launched successively. However, while this type of insecticide showed excellent insecticidal effects, it gradually exposed potential risks to environmental non-target organisms $[43,44]$. Broflanilide is a meta-diamide insecticide developed by Mitsui Chemicals and co-developed with BASF SE. Because of its novel mechanism of action, this product was expected to become a blockbuster product.

In view of these facts mentioned above, broflanilide was employed as the lead compound in this study. According to the principle of bioisosterism [45,46], we searched for the amide group of broflanilide in the 1,2,4-oxadiazole ring, replaced the benzene ring with a pyridine structure containing a thioether derivative, and designed (Figure 2) and synthesized (Scheme 1) a series of novel benzamides substituted with 1,2,4-oxadiazole. These new compounds were confirmed by ${ }^{1} \mathrm{H}$ NMR, ${ }^{13} \mathrm{C}$ NMR, and HRMS, and their insecticidal activities, fungicidal activities, and toxicity test of zebrafish embryo were studied. 
<smiles>Cc1cc(C(F)(F)C(F)(F)F)ccc1NC(=O)c1cccc(C)c1C(=O)NC(C)(C)CS(C)(=O)=O</smiles>

Flubendiamide<smiles>CNC(=O)c1cc(Cl)cc(C)c1NC(=O)c1cc(Br)nn1-c1ncccc1Cl</smiles>

Chlorantraniliprole<smiles>CNC(=O)c1cc(C#N)cc(C)c1NC(=O)c1cc(Br)nn1-c1ncccc1Cl</smiles>

Cyantraniliprole<smiles>CC(NC(=O)c1cc(Cl)cc(Br)c1NC(=O)c1cc(Br)nn1-c1ncccc1Cl)C1CC1</smiles>

Cyclaniliprole

Figure 1. Chemical structures of flubendiamide, chlorantraniliprole, cyantraniliprole, and cyclaniliprole.

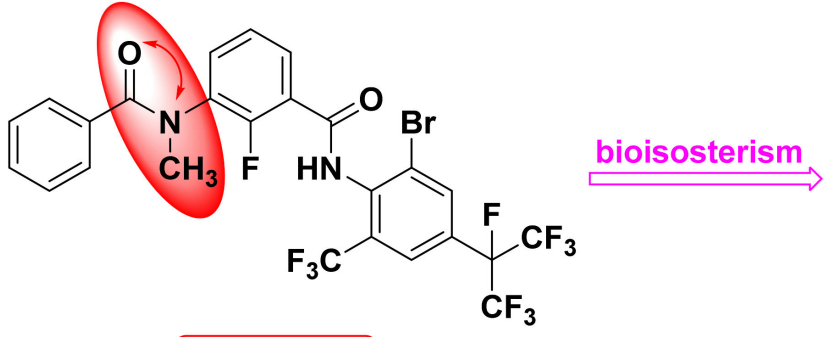

Broflanilide<smiles>[R]c1ccc(NC(=O)c2cc(-c3cccc(-c4nc(Cl)ccc4[R1])n3)ccc2Cl)cc1</smiles>

Target compounds

Figure 2. Design strategy of target compounds.<smiles>CCSc1ccc(Cl)nc1C(=O)OCCOC(=O)c1nc(Cl)ccc1SCCCCCCOC(=O)c1nc(Cl)ccc1Cl</smiles><smiles>COC(=O)c1cc(I)ccc1Cl</smiles>

4 5 6<smiles>CCSc1ccc(Cl)nc1-c1nc(-c2ccc(Cl)c(C(=O)OC)c2)no1</smiles><smiles>CCSc1ccc(Cl)nc1-c1nc(-c2ccc(Cl)c(C(=O)O)c2)no1</smiles><smiles>[CH]1CCC1</smiles>

7<smiles>[R]c1cccc(NC(=O)c2cc(-c3noc(-c4nc(Cl)ccc4S(=O)(=O)CC)n3)ccc2Cl)c1</smiles>

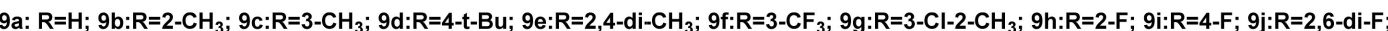

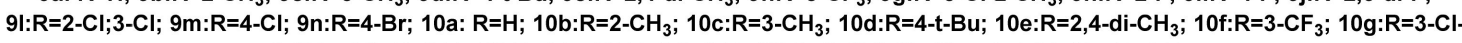
$2-\mathrm{CH}_{3} ; 10 \mathrm{~h}: \mathrm{R}=2-\mathrm{F} ; 10 \mathrm{i}: \mathrm{R}=4-\mathrm{F} ; 10 \mathrm{j}: \mathrm{R}=2,6-\mathrm{di}-\mathrm{F} ; 10 \mathrm{I}: \mathrm{R}=2-\mathrm{Cl} ; 3-\mathrm{Cl} ; 10 \mathrm{~m}: \mathrm{R}=4-\mathrm{Cl} ; 10 \mathrm{n}: \mathrm{R}=4-\mathrm{Br}$;

Scheme 1. Synthetic route of target compounds. Reagents and conditions: (a) DMS, $\mathrm{K}_{2} \mathrm{CO}_{3}$, acetone, reflux; (b) $\mathrm{CH}_{3} \mathrm{CH}_{2} \mathrm{SH}$, $\mathrm{KTB}, \mathrm{DMF}, 0-5{ }^{\circ} \mathrm{C}$, rt; (c) THF, $\mathrm{OH}^{-}$, reflux; (d) $\mathrm{CH}_{3} \mathrm{OH}, \mathrm{H}^{+}$, reflux; (e) $\mathrm{CuCN}$, L-proline, DMF, $100{ }^{\circ} \mathrm{C} ;(\mathbf{f}) \mathrm{NH}_{2} \mathrm{OH} \bullet \mathrm{HCl}$, $\mathrm{CH}_{3} \mathrm{CH}_{2} \mathrm{OH}$, rt; (g) $\mathrm{SOCl}_{2}$, reflux; $\mathrm{Et}_{3} \mathrm{~N}$, toluene, reflux; (h) THF, $\mathrm{OH}^{-}$, reflux; (i) $\mathrm{EDCI}, \mathrm{Et}_{3} \mathrm{~N}, \mathrm{CH}_{2} \mathrm{Cl}_{2}, \mathrm{rt}$; (j) mCPBA, $\mathrm{CH}_{2} \mathrm{Cl}_{2}$, rt. 


\section{Results and Discussion}

\subsection{Synthesis of Target Compounds}

The synthetic pathways to target compounds $\mathbf{9}$ and $\mathbf{1 0}$ are shown in Scheme 1. Intermediate I was prepared from 3,6-dichloropyridinecarboxylic acid $\mathbf{1}$ as a starting material via methylation, thioetherification, and hydrolysis reactions. During the thioetherification reaction, the newly prepared potassium ethanethiolate should be slowly added to the DMF solution of methyl 3,6-dichloropicolinate, and the temperature should be controlled at $0-5{ }^{\circ} \mathrm{C}$ to avoid the by-products (Scheme 2). As in our previous procedures [47], intermediate II was easily obtained. Methyl 2-chloro-5-(5-(6-chloro-3-(ethylthio) pyridin-2-yl)-1,2,4oxadiazol-3-yl) benzoate 7 was synthesized by cyclization reaction from imtermediate II and 6-chloro-3-(ethylthio)picolinoyl chloride that had been synthesized from intermediate I. Then, the compound 7 was hydrolyzed and spliced with substituted anilines to give a series of target compounds $\mathbf{9 a}-\mathbf{9 n}$. Finally, compound $\mathbf{9}$ was oxidized with mCPBA at room temperature to give target compound 10 , which avoided the impurities of pyridine$\mathrm{N}$-oxide. The characterization data for all synthesized compounds are provided in the supporting information file (Figures S1-S39).

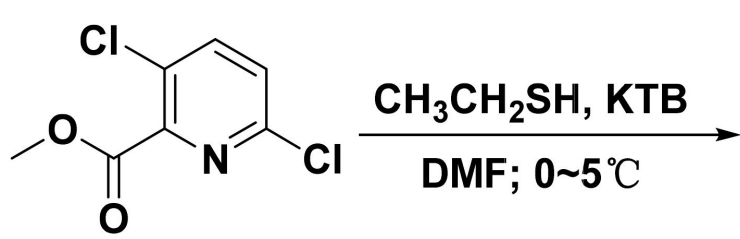

2<smiles>CCSc1ccc(Cl)nc1C(=O)OC</smiles>

3<smiles>[CH2+]OC(=O)c1nc(SCC)ccc1Cl</smiles>

by-product

Scheme 2. Synthesis of compound 3 via thioetherification.

\subsection{Spectrum Analysis of Target Compounds}

All the target compounds were confirmed by ${ }^{1} \mathrm{H}$ NMR, ${ }^{13} \mathrm{C}$ NMR, and HRMS. In the ${ }^{1} \mathrm{H}$ NMR spectra of $\mathbf{1 0 a}$, the $-\mathrm{NH}$ proton signals were found at $\delta 10.70 \mathrm{ppm}$. The signals of $\mathrm{CH}$ of the benzene and pyridine rings were assigned at $8.61-7.13 \mathrm{ppm}$. The signals at $\delta 3.78 \mathrm{ppm}$ and $1.26 \mathrm{ppm}$ were $-\mathrm{CH}_{2}$ and $-\mathrm{CH}_{3}$ peak, respectively. In the ${ }^{13} \mathrm{C}-\mathrm{NMR}$ spectra of compound 10a, the appearances of signals at 166.81 and $163.79 \mathrm{ppm}$ were assigned to the carbons of the 1,2,4-oxadiazole ring. Finally, all of the novel benzamides substituted with 1,2,4-oxadiazole exhibited a strong $[\mathrm{M}+\mathrm{H}]^{+}$peak in positive ion high-resolution electrospray mass spectra (HR-ESI-MS) analysis.

\subsection{Biological Activities of Target Compounds}

In Table 1, The target compounds had weak death rates against Mythimna sepatara $(5-40 \%)$ at $500 \mathrm{mg} / \mathrm{L}$, which were lower than the control drug broflanilide (100\%). In addition, all of the target compounds had good fungicidal activities at $100 \mathrm{mg} / \mathrm{L}$. Overall, the target compounds had better inhibitory activities against Botrytis cinereal than Fusarium graminearum, Marssonina mali, and Thanatephorus cucumeris. In particular, the inhibitory activity of compounds $10 \mathrm{a}(84.4 \%), \mathbf{1 0 d}(83.6 \%), \mathbf{1 0 e}(83.3 \%), \mathbf{1 0 f}(83.1 \%), \mathbf{1 0 i}(83.3 \%)$, and $101(83.6 \%)$ were better than pyraclostrobin $(81.4 \%)$, and $10 \mathrm{~b}(80.8 \%), 10 \mathrm{~g}(81.1 \%), \mathbf{1 0 h}$ $(81.4 \%)$, and $10 \mathrm{k}(81.9 \%)$ were comparable to pyraclostrobin. At the same time, compounds 9e $(57.7 \%), 9 f(63.5 \%), 9 g(65.9 \%), 9 h(57.1 \%)$, and 9k (61.2\%) also showed moderate activities. For Fusarium graminearum and Marssonina mali, compounds $\mathbf{9 a}-\mathbf{9 n}$ displayed weak inhibition, both of which were less than $25 \%$. Some of compound $\mathbf{1 0}$ had good activities (46.4-54.4\%) but were inferior to pyraclostrobin. For Thanatephorus cucumeris, compound $\mathbf{9}$ had no inhibitory activity, and some of compound $\mathbf{1 0}$ exhibited moderate inhibitory activities (37.5-50.3\%). From Table 2, we can see that compound 10 f had good inhibitory activity against Botrytis cinereal with $\mathrm{EC}_{50}$ of $14.44 \mu \mathrm{g} / \mathrm{mL}$. 
Table 1. Insecticidal and fungicidal activities of title compounds $\mathbf{9}$ and $\mathbf{1 0 .}$

\begin{tabular}{|c|c|c|c|c|c|c|}
\hline \multirow{2}{*}{ Compound } & \multirow{2}{*}{$\mathbf{R}$} & \multirow{2}{*}{$\begin{array}{c}\text { Insecticidal } \\
\text { Activities (500 mg/L, } \\
\text { Death Rates \%) } \\
\text { Mythimna sepatara }\end{array}$} & \multicolumn{4}{|c|}{ Fungicidal Activities (100 mg/L, Inhibition Rates \%) } \\
\hline & & & $\begin{array}{l}\text { Botrytis } \\
\text { cinereal }\end{array}$ & $\begin{array}{c}\text { Fusarium } \\
\text { graminearum }\end{array}$ & $\begin{array}{l}\text { Marssonina } \\
\text { mali }\end{array}$ & $\begin{array}{c}\text { Thanatephorus } \\
\text { cucumeris }\end{array}$ \\
\hline $9 a$ & $\mathrm{H}$ & 10 & 48.2 & 0 & 22.0 & 0 \\
\hline $9 b$ & $2-\mathrm{CH}_{3}$ & 5 & 25.0 & 2.4 & 15.3 & 0 \\
\hline 9c & $4-\mathrm{CH}_{3}$ & 20 & 48.2 & 0.9 & 13.5 & 0 \\
\hline $9 d$ & 4-t-Bu & 0 & 18.5 & 18.2 & 16.5 & 0 \\
\hline $9 e$ & 2,4-di- $\mathrm{CH}_{3}$ & 30 & 57.7 & 2.9 & 10.6 & 0 \\
\hline $9 f$ & $3-\mathrm{CF}_{3}$ & 25 & 63.5 & 8.5 & 16.5 & 0 \\
\hline $9 g$ & $3-\mathrm{Cl}-2-\mathrm{CH}_{3}$ & 15 & 65.9 & 0 & 10.0 & 0 \\
\hline $9 \mathrm{~h}$ & $2-\mathrm{F}$ & 30 & 57.1 & 5.3 & 20.9 & 0 \\
\hline $9 \mathrm{i}$ & 4-F & 10 & 23.5 & 6.8 & 16.8 & 0 \\
\hline $9 j$ & 2,6-di-F & 0 & 19.7 & 16.2 & 21.5 & 0 \\
\hline $9 \mathrm{k}$ & 2-Cl & 5 & 61.2 & 15.0 & 14.4 & 0 \\
\hline 91 & $3-\mathrm{Cl}$ & 0 & 20.9 & 17.4 & 24.4 & 0 \\
\hline $9 m$ & $4-\mathrm{Cl}$ & 5 & 5.3 & 0 & 12.4 & 0 \\
\hline $9 n$ & $4-\mathrm{Br}$ & 25 & 17.7 & 4.7 & 18.5 & 0 \\
\hline $10 a$ & $\mathrm{H}$ & 20 & 84.4 & 25.0 & 39.2 & 0 \\
\hline $10 b$ & $2-\mathrm{CH}_{3}$ & 15 & 80.8 & 29.4 & 37.8 & 0 \\
\hline $10 c$ & $4-\mathrm{CH}_{3}$ & 10 & 22.9 & 53.1 & 34.1 & 0 \\
\hline $10 \mathrm{~d}$ & 4-t-Bu & 25 & 83.6 & 52.5 & 44.2 & 44.2 \\
\hline $10 e$ & 2,4-di- $\mathrm{CH}_{3}$ & 35 & 83.3 & 46.4 & 39.4 & 41.9 \\
\hline $10 f$ & $3-\mathrm{CF}_{3}$ & 40 & 83.1 & 43.6 & 45.8 & 37.5 \\
\hline $10 \mathrm{~g}$ & 3-Cl-2-CH & 25 & 81.1 & 50.0 & 42.8 & 44.7 \\
\hline $10 \mathrm{~h}$ & $2-\mathrm{F}$ & 40 & 81.4 & 51.4 & 48.3 & 50.3 \\
\hline $10 \mathrm{i}$ & 4-F & 25 & 83.3 & 54.4 & 43.1 & 44.2 \\
\hline $10 \mathrm{j}$ & 2,6-di-F & 15 & 31.8 & 23.2 & 38.8 & 0 \\
\hline $10 \mathrm{k}$ & $2-\mathrm{Cl}$ & 5 & 81.9 & 50.3 & 45.0 & 46.1 \\
\hline 101 & $3-\mathrm{Cl}$ & 15 & 83.6 & 48.1 & 39.2 & 44.4 \\
\hline $10 \mathrm{~m}$ & $4-\mathrm{Cl}$ & 15 & 22.7 & 13.2 & 33.2 & 0 \\
\hline $10 n$ & $4-\mathrm{Br}$ & 20 & 17.9 & 11.8 & 37.1 & 0 \\
\hline Broflanilide & & 100 & nt & nt & nt & nt \\
\hline Chlorantraniliprole & & 100 & nt & nt & $\mathrm{nt}$ & nt \\
\hline Pyraclostrobin & & nt & 81.4 & 100 & 84.1 & 100 \\
\hline
\end{tabular}

Note: $\mathrm{nt}=$ not tested. All the data were determined three times.

Table 2. $\mathrm{EC}_{50}$ of compounds $10 \mathrm{f}$ to Botrytis cinereal.

\begin{tabular}{cccc}
\hline Compound & $\mathrm{y}=\mathbf{a}+\mathbf{b x}$ & $\boldsymbol{r}^{\mathbf{2}}$ & $\mathrm{EC}_{50} /\left(\boldsymbol{\mu g} \mathbf{~ m L}^{-\mathbf{1}}\right)$ \\
\hline $\mathbf{1 0 f}$ & $\mathrm{y}=1.5374 \mathrm{x}+3.2171$ & 0.9961 & 14.44 \\
\hline
\end{tabular}

According to the insecticidal activity result, we speculated that the amide bridge bond played a key role in maintaining insecticidal activity, and it was likely that it would interact with the receptor through its hydrogen bond. Searching the amide bridge bond in 1,2,4-oxadiazole, we found that it lacked the corresponding hydrogen bond, and the group bulk increased, which resulted in the blocking of the binding of the compounds to the receptor and did not show a good insecticidal activity. It could be seen from Table 1 that the fungicidal activities of compound $\mathbf{1 0}$ was significantly higher than compound $\mathbf{9}$, indicating that the structure containing ethylsulfonyl was beneficial in increasing the activity. The SAR of compound 10 in terms of fungicidal activities (Table 1) was that when there was no substituent on the benzene ring, the activity against Botrytis cinereal was superior to other compounds. In addition, by comparing the control efficacy of compounds 10k, 101, $10 \mathrm{~m}, 10 \mathrm{c}$, and 10n, we found that they showed that the para-position of aniline-containing substituents was not conducive to improving the activity. 


\subsection{Toxicity to Zebrafish Embryo}

According to the fungicidal activity result, we selected compounds 9 f and $10 f$ with better activity to study the lethal and teratogenic effects exposure on zebrafish embryos from 6 to $96 \mathrm{hpf}$ (Figure 3). When the $9 \mathrm{f}$ concentration exceeded $2 \mathrm{mg} / \mathrm{L}$, the mortality rate increased sharply. At $10 \mathrm{mg} / \mathrm{L}$, the mortality rate reached as high as $90 \%$. The resulting $\mathrm{LC}_{50}$ value for compound $9 \mathrm{f}$ was $5.26 \mathrm{mg} / \mathrm{L}$ (Figure $3 \mathrm{~A}$ ). Similarly, the mortality rate of $10 \mathrm{f}$ showed concentration-dependent curves (Figure 3) with a $\mathrm{LC}_{50}$ value of $20.58 \mathrm{mg} / \mathrm{L}$ (Figure 3B). Moreover, $9 \mathrm{f}$ and 10f produced similar teratogenic and decreased hatching effects on zebrafish embryos at $72 \mathrm{hpf}$ (Figure 3C-F). At $72 \mathrm{hpf}$, the hatching rates of the compounds 9 f and $10 \mathrm{f}$ under $10 \mathrm{mg} / \mathrm{L}$ exposure were about $43 \%$ and $82 \%$, respectively. In addition, the malformation rate of $9 \mathrm{f}$ was significantly higher than $\mathbf{1 0 f}$ at the same concentration.

A

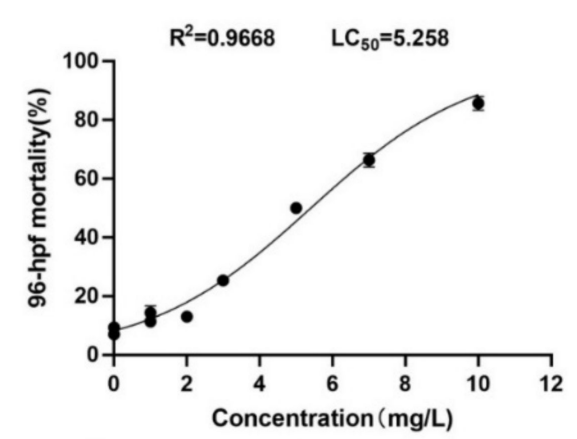

$\mathrm{C}$

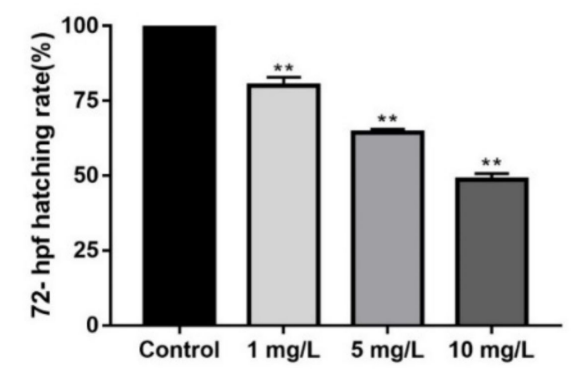

$\mathrm{E}$

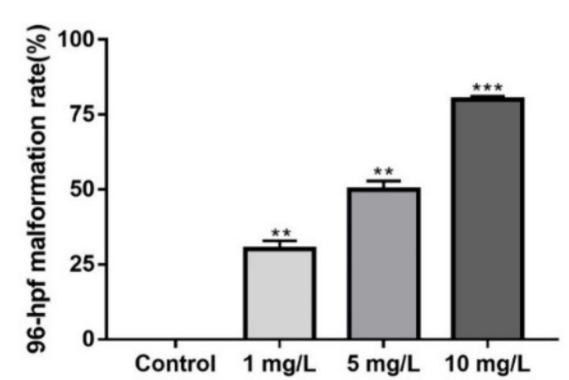

B
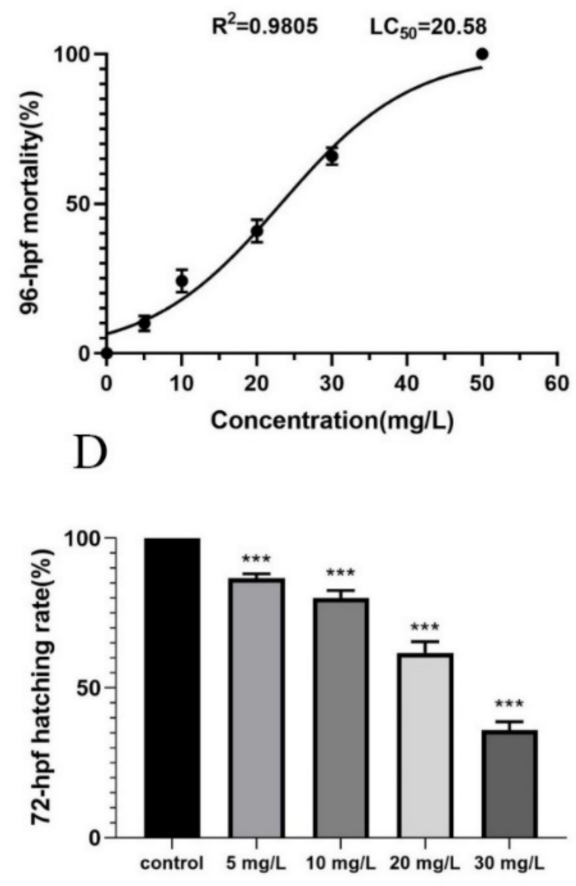

F

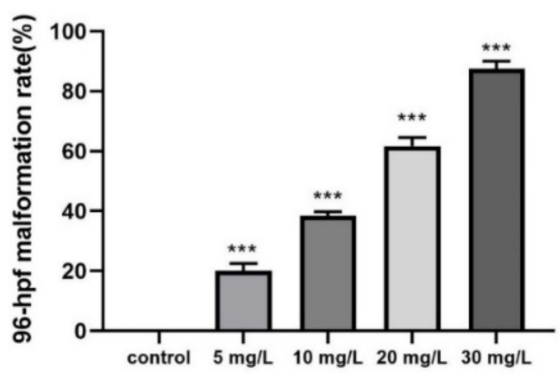

Figure 3. Zebrafish embryo mortality, hatching rate, and malformation rates after exposure to compounds $\mathbf{9 f}(\mathbf{A}, \mathbf{C}, \mathbf{E})$ and $\mathbf{1 0 f}(\mathbf{B}, \mathbf{D}, \mathbf{F})$. Note: "**" represents significant differences at ${ }^{* *} p \leq 0.01$ and ${ }^{* * *} p \leq 0.001$ by one-way ANOVA followed by Dunnett's test.

As the time and concentration increased, zebrafish embryos showed obvious developmental delay. At 76-96 hpf, a series of malformations appeared, such as delayed yolk absorption, pericardial cyst, lack of melanin, yolk sac, and bent spine (Figure 4). Among 
them, yolk cyst was the most obvious. By comparing the lethal and teratogenic effects of $9 f$ and $10 f$ exposure on zebrafish embryos, we were able to find that the toxicity of $9 \mathrm{f}$ to zebrafish embryos was higher than that of $\mathbf{1 0 f}$. Thus, we speculated that the structure containing ethylsulfonyl was beneficial to reduce the toxicity to zebrafish embryos.
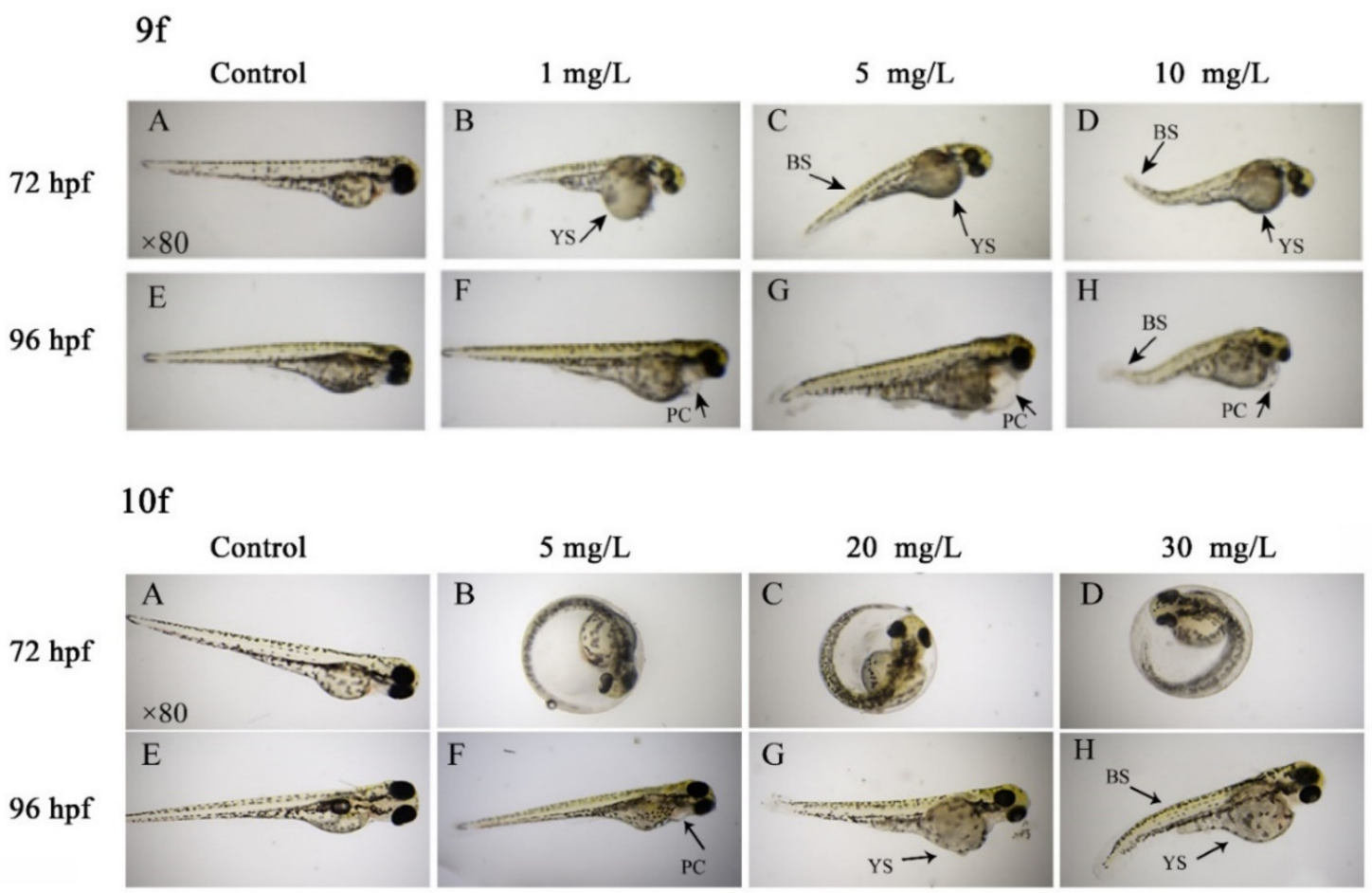

Figure 4. Zebrafish embryo malformation after exposure to compounds 9f and 10f. Note: PC, pericardial cyst; YS, yolk sac; BS, bent spine.

\section{Experimental Section}

\subsection{General Information}

Melting points were determined using an X-4 apparatus (Taike, Beijing, China) and the thermometer was uncorrected. ${ }^{1} \mathrm{H}$ NMR and ${ }^{13} \mathrm{C}$ NMR spectra were measured on BRUKER Avance $500 \mathrm{MHz}$ spectrometer (Bruker $500 \mathrm{MHz}$, Fallanden, Switzerland) using $\mathrm{CDCl}_{3}$ or DMSO as the solvent. High-resolution electrospray mass spectra (HR-ESI-MS) were determined using an UPLC H CLASS/QTOF G2 XS mass spectrometer (Waters, Milford, CT, USA). All the reagents were analytical grade or synthesized in our laboratory.

Ethics statement: The Institutional Animal Care and Use Committee (IACUC) at Wenzhou Medical University (SYXK 2019-0009, April 4, 2019 to April 4, 2024) approved our study plan for proper use of zebrafish. All studies were carried out in strict accordance with the guidelines of the IACUC. All dissection was performed on ice, and all efforts were made to minimize suffering.

\subsection{Synthesis}

\subsubsection{Synthesis of Intermediate I}

Methyl 3,6-dichloropicolinate (2): To a stirred solution of 3,6-dichloropicolinic acid (1) $(0.10 \mathrm{mmol})$ in acetone $(10 \mathrm{~mL})$, we added DMS $(1.3 \mathrm{~g}, 0.01 \mathrm{~mol})$ and $\mathrm{K}_{2} \mathrm{CO}_{3}(1.7 \mathrm{~g})$. After stirring at $40^{\circ} \mathrm{C}$ for $8 \mathrm{~h}$, the mixture was cooled to room temperature and poured into water, the precipitation was filtered and dried to give $1.8 \mathrm{~g}$ light yellow solid. Yield: 90.1\%, m.p. $54-55{ }^{\circ} \mathrm{C}$ ([48], 53-54 $\left.{ }^{\circ} \mathrm{C}\right) ;{ }^{1} \mathrm{H}$ NMR (500 MHz, Chloroform-d) $\delta 7.77$ $(\mathrm{d}, J=8.5 \mathrm{~Hz}, 1 \mathrm{H}), 7.42(\mathrm{~d}, J=8.5 \mathrm{~Hz}, 1 \mathrm{H}), 4.00(\mathrm{~s}, 3 \mathrm{H})$. 
Methyl 6-chloro-3-(ethylthio) picolinate (3): KTB (1.4 g), DMF (30 mL), and ethyl mercaptan $(2.5 \mathrm{~g}, 0.04 \mathrm{~mol})$ were added to a three-necked flask and reacted at $0{ }^{\circ} \mathrm{C}$ for about $1 \mathrm{~h}$ to give $\mathrm{CH}_{3} \mathrm{CH}_{2} \mathrm{SK}$. The $\mathrm{CH}_{3} \mathrm{CH}_{2} \mathrm{SK}$ was dropped into a stirred solution of compound $2(3.7 \mathrm{~g})$ in DMF $(20 \mathrm{~mL})$ at $0{ }^{\circ} \mathrm{C}$. After stirring at room temperature for $2 \mathrm{~h}$, the mixture was quenched with water and extracted by EtOAc $(100 \mathrm{~mL})$. The extraction was dried over anhydrous $\mathrm{MgSO}_{4}$ and filtered. The filtration was concentrated and separated by column chromatography to give $3.1 \mathrm{~g}$ yellow solid. Yield: $74.5 \%$, m.p. $128-129{ }^{\circ} \mathrm{C} ;{ }^{1} \mathrm{H}$ NMR $(500 \mathrm{MHz}$, Chloroform- $d) \delta 7.66(\mathrm{~d}, J=8.5 \mathrm{~Hz}, 1 \mathrm{H}), 7.41(\mathrm{~d}, J=8.5 \mathrm{~Hz}, 1 \mathrm{H}), 4.00(\mathrm{~s}, 3 \mathrm{H}), 2.95$ $(\mathrm{q}, J=7.5 \mathrm{~Hz}, 2 \mathrm{H}), 1.39(\mathrm{t}, J=7.5 \mathrm{~Hz}, 3 \mathrm{H})$.

Intermediate I: $30 \% \mathrm{NaOH}(5 \mathrm{~mL})$ was added to a solution of compound $3(3.3 \mathrm{~g})$ in THF. The mixture was then refluxed for $3 \mathrm{~h}$. Afterwards, the mixture was cooled to room temperature and poured into water. Then, we adjusted the $\mathrm{pH}$ to $2-3$, and $2.8 \mathrm{~g}$ white solid precipitate was obtained. Yield: $92.1 \%$, m.p. $119-120{ }^{\circ} \mathrm{C} ;{ }^{1} \mathrm{H}$ NMR $(500 \mathrm{MHz}$, Chloroform- $d$ ) $\delta 10.76(\mathrm{~s}, 1 \mathrm{H}), 7.71(\mathrm{~d}, J=8.5 \mathrm{~Hz}, 1 \mathrm{H}), 7.49(\mathrm{~d}, J=8.5 \mathrm{~Hz}, 1 \mathrm{H}), 2.97(\mathrm{q}, J=7.5 \mathrm{~Hz}, 2 \mathrm{H})$, $1.44(\mathrm{t}, 3 \mathrm{H})$.

\subsubsection{Synthesis of Intermediate II}

The synthesis of intermediate II refers to our previous work.

3.2.3. Methyl 2-Chloro-5-(5-(6-Chloro-3-(Ethylthio) Pyridin-2-yl)-1,2,4-Oxadiazol-3-yl) Benzoate (7)

The intermediate $\mathbf{I}(1.1 \mathrm{~g}, 5 \mathrm{mmol})$ and $\mathrm{SOCl}_{2}(20 \mathrm{~mL})$ were added to a $100 \mathrm{~mL}$ flask and the mixture was refluxed for $6 \mathrm{~h}$. Then, $\mathrm{SOCl}_{2}$ was removed under reduced pressure to give 6-chloro-3-(ethylthio)picolinoyl chloride.

To a solution of intermediate II $(1.1 \mathrm{~g}, 5.5 \mathrm{mmol})$ and triethylamine $(1.2 \mathrm{~g}, 12 \mathrm{mmol})$ in toluene $(100 \mathrm{~mL})$, we added the prepared 6-chloro-3-(ethylthio)picolinoyl chloride dropwise at $0{ }^{\circ} \mathrm{C}$ for $1 \mathrm{~h}$. The mixture was then refluxed for $2 \mathrm{~h}$. Afterwards, the mixture was cooled to room temperature and washed by saturated sodium chloride solution $(100 \mathrm{~mL} \times 3)$. The organic layer was dried by $\mathrm{Na}_{2} \mathrm{SO}_{4}$ and removed to give yellow solid (1.4 g). Yield: 62.2\%, m.p. $155-157^{\circ} \mathrm{C} ;{ }^{1} \mathrm{H}$ NMR (500 MHz, Chloroform- $d$ ) $\delta 8.63$ $(\mathrm{d}, J=2.0 \mathrm{~Hz}, 1 \mathrm{H}), 8.35-8.20(\mathrm{~m}, 1 \mathrm{H}), 7.89(\mathrm{~d}, J=8.5 \mathrm{~Hz}, 1 \mathrm{H}), 7.66(\mathrm{~d}, J=8.0 \mathrm{~Hz}, 1 \mathrm{H}), 7.54$ $(\mathrm{d}, J=8.5 \mathrm{~Hz}, 1 \mathrm{H}), 3.87(\mathrm{~s}, 3 \mathrm{H}), 3.17(\mathrm{q}, \mathrm{J}=7.0 \mathrm{~Hz}, 2 \mathrm{H}), 1.32(\mathrm{t}, \mathrm{J}=7.5 \mathrm{~Hz}, 3 \mathrm{H}) ;$ HRMS calcd for $\mathrm{C}_{17} \mathrm{H}_{14} \mathrm{Cl}_{2} \mathrm{~N}_{3} \mathrm{O}_{3} \mathrm{~S}[\mathrm{M}+\mathrm{H}]^{+} 410.0127$, found 410.0126 .

3.2.4. 2-Chloro-5-(5-(6-Chloro-3-(Ethylthio) Pyridin-2-yl)-1,2,4-Oxadiazol-3-yl) Benzoic Acid (8)

We added $30 \% \mathrm{NaOH}(5 \mathrm{~mL})$ to a solution of compound $7(0.8 \mathrm{~g}, 2.0 \mathrm{mmol})$ in THF. The mixture was then refluxed for $2 \mathrm{~h}$. Afterwards, the mixture was cooled to room temperature and the solvent was removed. Then, we adjusted the $\mathrm{pH}$ to $2-3$, and white solid precipitate was obtained (0.7 g). Yield: $90.8 \%$, m.p. $225-227{ }^{\circ} \mathrm{C} ;{ }^{1} \mathrm{H}$ NMR $(500 \mathrm{MHz}$, Chloroform- $d)$ $\delta 13.47(\mathrm{~s}, 1 \mathrm{H}), 8.55(\mathrm{~d}, J=2.0 \mathrm{~Hz}, 1 \mathrm{H}), 8.36(\mathrm{~d}, J=8.5 \mathrm{~Hz}, 1 \mathrm{H}), 8.26-8.19(\mathrm{~m}, 1 \mathrm{H}), 7.91(\mathrm{~d}$, $J=8.5 \mathrm{~Hz}, 1 \mathrm{H}), 7.80(\mathrm{~d}, J=8.0 \mathrm{~Hz}, 1 \mathrm{H}), 3.16(\mathrm{q}, \mathrm{J}=7.5 \mathrm{~Hz}, 2 \mathrm{H}), 1.30(\mathrm{t}, \mathrm{J}=7.0 \mathrm{~Hz}, 3 \mathrm{H})$; HRMS calcd for $\mathrm{C}_{16} \mathrm{H}_{12} \mathrm{Cl}_{2} \mathrm{~N}_{3} \mathrm{O}_{3} \mathrm{~S}[\mathrm{M}+\mathrm{H}]^{+}$395.9971, found 395.9973.

\subsubsection{Synthesis of Target Compound 9}

To a solution of compound $8(1.0 \mathrm{~g}, 2.0 \mathrm{mmol})$, EDCI $(0.1 \mathrm{~g})$ and triethylamine $(0.2 \mathrm{~g})$ in DCM $(100 \mathrm{~mL})$, we added the substituted aniline $(3.0 \mathrm{mmol})$ at $0{ }^{\circ} \mathrm{C}$, and the mixture was stirred for $8 \mathrm{~h}$ to give compound 9 by the method of column chromatography separation.

2-Chloro-5-(5-(6-chloro-3-(ethylthio)pyridin-2-yl)-1,2,4-oxadiazol-3-yl)-N-phenylbenzamide (9a): White solid, yield 73.4\%, m.p. $243-245{ }^{\circ} \mathrm{C} ;{ }^{1} \mathrm{H}$ NMR (500 MHz, DMSO- $\left.d_{6}\right){ }^{1} \mathrm{H}$ NMR $(500 \mathrm{MHz}$, Chloroform- $d$ ) $\delta 10.70(\mathrm{~s}, 1 \mathrm{H}), 8.23-8.18(\mathrm{~m}, 2 \mathrm{H}), 8.13(\mathrm{~d}, J=9.0 \mathrm{~Hz}, 1 \mathrm{H})$, $7.88-7.84(\mathrm{~m}, 1 \mathrm{H}), 7.79(\mathrm{~d}, J=8.5 \mathrm{~Hz}, 1 \mathrm{H}), 7.73(\mathrm{~d}, J=8.0 \mathrm{~Hz}, 2 \mathrm{H}), 7.38(\mathrm{t}, J=8.0 \mathrm{~Hz}, 2 \mathrm{H}), 7.14$ $(\mathrm{t}, J=7.0 \mathrm{~Hz}, 1 \mathrm{H}), 3.15(\mathrm{q}, J=7.5 \mathrm{~Hz}, 2 \mathrm{H}), 1.30(\mathrm{t}, J=7.0 \mathrm{~Hz}, 3 \mathrm{H}) ;{ }^{13} \mathrm{C}$ NMR $(500 \mathrm{MHz}$, DMSO- $d_{6}$ ) $\delta 172.43,167.11,164.10,145.88,138.71,138.64,138.22,138.13,137.88,135.87$, 
133.66, 131.22, 129.60, 129.01, 127.81, 127.29, 124.30, 119.89, 25.53, 13.36; HRMS calcd for $\mathrm{C}_{22} \mathrm{H}_{17} \mathrm{Cl}_{2} \mathrm{~N}_{4} \mathrm{O}_{2} \mathrm{~S}[\mathrm{M}+\mathrm{H}]^{+}$471.0444, found 471.0447 .

2-Chloro-5-(5-(6-chloro-3-(ethylthio)pyridin-2-yl)-1,2,4-oxadiazol-3-yl)-N-(o-tolyl)benzamide (9b): White solid, yield $77.4 \%$, m.p. $230-232{ }^{\circ} \mathrm{C} ;{ }^{1} \mathrm{H}$ NMR (500 MHz, DMSO- $d_{6}$ ) $\delta 10.19$ (s, $1 \mathrm{H}), 8.26(\mathrm{~d}, J=2.0 \mathrm{~Hz}, 1 \mathrm{H}), 8.20(\mathrm{dd}, J=8.5,2.0 \mathrm{~Hz}, 1 \mathrm{H}), 8.14(\mathrm{~d}, J=9.0 \mathrm{~Hz}, 1 \mathrm{H}), 7.86(\mathrm{~d}$, $J=8.5 \mathrm{~Hz}, 1 \mathrm{H}), 7.80(\mathrm{~d}, J=8.5 \mathrm{~Hz}, 1 \mathrm{H}), 7.49(\mathrm{~d}, J=7.5 \mathrm{~Hz}, 1 \mathrm{H}), 7.29(\mathrm{~d}, J=7.5 \mathrm{~Hz}, 1 \mathrm{H})$, $7.25(\mathrm{t}, J=6.5 \mathrm{~Hz}, 1 \mathrm{H}), 7.19(\mathrm{t}, J=7.5 \mathrm{~Hz}, 1 \mathrm{H}), 3.17(\mathrm{q}, J=7.0 \mathrm{~Hz}, 2 \mathrm{H}), 2.30(\mathrm{~s}, 3 \mathrm{H}), 1.32$ $(\mathrm{t}, J=7.5 \mathrm{~Hz}, 3 \mathrm{H}) ;{ }^{13} \mathrm{C}$ NMR $\left(500 \mathrm{MHz}\right.$, DMSO- $\left.d_{6}\right){ }^{13} \mathrm{C}$ NMR $\left(126 \mathrm{MHz}\right.$, DMSO- $\left.d_{6}\right) \delta 172.45$, $166.07,163.92$, 145.90, 138.67, 137.96, 137.61, 136.24, 133.69, 133.53, 133.38, 132.91, 131.23, 130.94, 129.56, 129.38, 127.83, 127.29, 125.25, 119.91, 25.55, 20.64, 13.38; HRMS calcd for $\mathrm{C}_{23} \mathrm{H}_{19} \mathrm{Cl}_{2} \mathrm{~N}_{4} \mathrm{O}_{2} \mathrm{~S}[\mathrm{M}+\mathrm{H}]^{+} 485.0600$, found 485.0600 .

2-Chloro-5-(5-(6-chloro-3-(ethylthio)pyridin-2-yl)-1,2,4-oxadiazol-3-yl)-N-(p-tolyl)benzamide (9c): White solid, yield 74.3\%, m.p. $251-253{ }^{\circ} \mathrm{C} ;{ }^{1} \mathrm{H}$ NMR (500 MHz, DMSO- $\left.d_{6}\right) \delta 10.61$ (s, $1 \mathrm{H}), 8.19(\mathrm{~m}, 2 \mathrm{H}), 8.13(\mathrm{~d}, J=9.0 \mathrm{~Hz}, 1 \mathrm{H}), 7.85(\mathrm{~d}, J=8.5 \mathrm{~Hz}, 1 \mathrm{H}), 7.79(\mathrm{~d}, J=9.0 \mathrm{~Hz}, 1 \mathrm{H})$, $7.61(\mathrm{~d}, J=8.0 \mathrm{~Hz}, 2 \mathrm{H}), 7.18(\mathrm{~d}, J=8.0 \mathrm{~Hz}, 2 \mathrm{H}), 3.16(\mathrm{~d}, J=7.5 \mathrm{~Hz}, 2 \mathrm{H}), 2.29(\mathrm{~s}, 3 \mathrm{H}), 1.30$ $(\mathrm{t}, J=7.5 \mathrm{~Hz}, 3 \mathrm{H}) ;{ }^{13} \mathrm{C}$ NMR $\left(500 \mathrm{MHz}, \mathrm{DMSO}-d_{6}\right) \delta 172.46,167.15,163.96,145.90$, $138.63,138.29,137.98,137.57,136.26,133.71,133.41,131.25,129.58,129.41,127.85,127.32$, 125.02, 119.93, 25.56, 20.67, 13.39; HRMS calcd for $\mathrm{C}_{23} \mathrm{H}_{19} \mathrm{Cl}_{2} \mathrm{~N}_{4} \mathrm{O}_{2} \mathrm{~S}[\mathrm{M}+\mathrm{H}]^{+} 485.0600$, found 485.0606 .

N-(4-(tert-Butyl) phenyl)-2-chloro-5-(5-(6-chloro-3-(ethylthio) pyridin-2-yl)-1,2,4-oxadiazol3-yl) benzamide (9d): White solid, yield $78.4 \%$,. m.p. $243-244{ }^{\circ} \mathrm{C} ;{ }^{1} \mathrm{H}$ NMR (500 MHz, DMSO$\left.d_{6}\right) \delta 10.63(\mathrm{~s}, 1 \mathrm{H}), 8.23-8.17(\mathrm{~m}, 2 \mathrm{H}), 8.14(\mathrm{~d}, J=9.0 \mathrm{~Hz}, 1 \mathrm{H}), 7.85(\mathrm{~d}$, $J=8.0 \mathrm{~Hz}, 1 \mathrm{H}), 7.79(\mathrm{~d}, J=8.5 \mathrm{~Hz}, 1 \mathrm{H}), 7.64(\mathrm{~d}, J=8.5 \mathrm{~Hz}, 2 \mathrm{H}), 7.39(\mathrm{~d}, J=8.5 \mathrm{~Hz}, 2 \mathrm{H}), 3.16$ $(\mathrm{q}, J=7.5 \mathrm{~Hz}, 2 \mathrm{H}), 1.34-1.26(\mathrm{~m}, 12 \mathrm{H}) ;{ }^{13} \mathrm{C}$ NMR $\left(500 \mathrm{MHz}, \mathrm{DMSO}-d_{6}\right) \delta 172.46,167.40$, $167.19,138.54,138.11,135.64,133.72,133.30,133.04,133.00,131.21,127.88,127.53,126.84$, $126.17,124.98,25.64,20.70,18.01,13.34$; HRMS calcd for $\mathrm{C}_{26} \mathrm{H}_{25} \mathrm{Cl}_{2} \mathrm{~N}_{4} \mathrm{O}_{2} \mathrm{~S}[\mathrm{M}+\mathrm{H}]^{+}$ 527.1070 , found 527.1071 .

2-Chloro-5-(5-(6-chloro-3-(ethylthio)pyridin-2-yl)-1,2,4-oxadiazol-3-yl)-N-(2,4-dimethylphenyl) benzamide (9e): Grey solid, yield $69.2 \%$, m.p. $255-256{ }^{\circ} \mathrm{C} ;{ }^{1} \mathrm{H}$ NMR $\left(500 \mathrm{MHz}, \mathrm{DMSO}-d_{6}\right) \delta$ $10.10(\mathrm{~s}, 1 \mathrm{H}), 8.24(\mathrm{~d}, J=2.0 \mathrm{~Hz}, 1 \mathrm{H}), 8.19(\mathrm{dd}, J=8.0,2.0 \mathrm{~Hz}, 1 \mathrm{H}), 8.14(\mathrm{~d}, J=8.5 \mathrm{~Hz}, 1 \mathrm{H})$, $7.83(\mathrm{dd}, J=24.0,8.5 \mathrm{~Hz}, 2 \mathrm{H}), 7.34(\mathrm{~d}, J=8.0 \mathrm{~Hz}, 1 \mathrm{H}), 7.10(\mathrm{~s}, 1 \mathrm{H}), 7.05(\mathrm{~d}, J=8.0 \mathrm{~Hz}, 1 \mathrm{H})$, $3.17(\mathrm{q}, J=7.0 \mathrm{~Hz}, 2 \mathrm{H}), 2.28(\mathrm{~d}, J=6.0 \mathrm{~Hz}, 6 \mathrm{H}), 1.32(\mathrm{t}, J=7.5 \mathrm{~Hz}, 3 \mathrm{H}) ;{ }^{13} \mathrm{C}$ NMR $(500 \mathrm{MHz}$, DMSO- $\left.d_{6}\right) \delta 172.46,167.40,167.19,138.54,138.11,135.64,133.72,133.30,133.04,131.21$, $127.88,127.53,126.84,126.17,25.64,20.70,18.01,13.34 ; \mathrm{HRMS}$ calcd for $\mathrm{C}_{24} \mathrm{H}_{21} \mathrm{Cl}_{2} \mathrm{~N}_{4} \mathrm{O}_{2} \mathrm{~S}$ $[\mathrm{M}+\mathrm{H}]^{+} 499.0757$, found 499.0763 .

2-Chloro-5-(5-(6-chloro-3-(ethylthio)pyridin-2-yl)-1,2,4-oxadiazol-3-yl)-N-(3-(trifluoromethyl) phenyl)benzamide (9f): White solid, yield 77.8\%, m.p. $211-214{ }^{\circ} \mathrm{C} ;{ }^{1} \mathrm{H}$ NMR $(500 \mathrm{MHz}$, DMSO- $\left.d_{6}\right) \delta 11.06(\mathrm{~s}, 1 \mathrm{H}), 8.27(\mathrm{~d}, J=2.0 \mathrm{~Hz}, 1 \mathrm{H}), 8.25-8.21(\mathrm{~m}, 2 \mathrm{H}), 8.14(\mathrm{~d}, J=9.0 \mathrm{~Hz}$, $1 \mathrm{H}), 7.92(\mathrm{~d}, J=8.0 \mathrm{~Hz}, 1 \mathrm{H}), 7.89(\mathrm{~d}, J=8.5 \mathrm{~Hz}, 1 \mathrm{H}), 7.80(\mathrm{~d}, J=9.0 \mathrm{~Hz}, 1 \mathrm{H}), 7.64(\mathrm{t}$, $J=8.0 \mathrm{~Hz}, 1 \mathrm{H}), 7.51(\mathrm{~d}, J=8.0 \mathrm{~Hz}, 1 \mathrm{H}), 3.16(\mathrm{q}, J=7.5 \mathrm{~Hz}, 2 \mathrm{H}), 1.30(\mathrm{t}, J=7.0 \mathrm{~Hz}, 3 \mathrm{H}) ;{ }^{13} \mathrm{C}$ NMR (500 MHz, DMSO-d $\left.d_{6}\right) \delta 172.48,167.10,164.59,145.91,139.49,138.68,138.63,138.59$, $138.28,137.58,137.35,133.70,131.35,131.29,130.37,127.88,127.81,125.11,123.53,120.68$, 116.00, 25.57, 13.36; HRMS calcd for $\mathrm{C}_{23} \mathrm{H}_{16} \mathrm{Cl}_{2} \mathrm{~F}_{3} \mathrm{~N}_{4} \mathrm{O}_{2} \mathrm{~S}[\mathrm{M}+\mathrm{H}]^{+} 539.0318$, found 539.0322 .

2-Chloro-N-(3-chloro-2-methylphenyl)-5-(5-(6-chloro-3-(ethylthio) pyridin-2-yl)-1,2,4oxadiazol-3-yl) benzamide (9g): Yellow solid, yield 79.7\%. m.p. $225-226{ }^{\circ} \mathrm{C} ;{ }^{1} \mathrm{H}$ NMR $\left(500 \mathrm{MHz}\right.$, DMSO- $\left.d_{6}\right) \delta 10.44(\mathrm{~s}, 1 \mathrm{H}), 8.29(\mathrm{~d}, J=1.5 \mathrm{~Hz}, 1 \mathrm{H}), 8.21(\mathrm{dd}, J=8.0,1.5 \mathrm{~Hz}$, $1 \mathrm{H}), 8.14(\mathrm{~d}, J=9.0 \mathrm{~Hz}, 1 \mathrm{H}), 7.87(\mathrm{~d}, J=8.0 \mathrm{~Hz}, 1 \mathrm{H}), 7.80(\mathrm{~d}, J=9.0 \mathrm{~Hz}, 1 \mathrm{H}), 7.47(\mathrm{~d}$, $J=8.0 \mathrm{~Hz}, 1 \mathrm{H}), 7.39(\mathrm{~d}, J=8.0 \mathrm{~Hz}, 1 \mathrm{H}), 7.29(\mathrm{t}, J=8.0 \mathrm{~Hz}, 1 \mathrm{H}), 3.16(\mathrm{q}, J=7.5 \mathrm{~Hz}, 2 \mathrm{H}), 2.35$ $(\mathrm{s}, 3 \mathrm{H}), 1.32(\mathrm{t}, J=7.5 \mathrm{~Hz}, 3 \mathrm{H}) ;{ }^{13} \mathrm{C}$ NMR $\left(500 \mathrm{MHz}, \mathrm{DMSO}-d_{6}\right) \delta 172.39,167.05,164.53$, $145.85,138.65$, 138.24, 137.61, 137.10, 134.00, 133.62, 131.53, 131.19, 129.61, 127.77, 127.40, $127.18,127.14,127.08,125.45,124.97,25.54,15.30,13.26$; HRMS calcd for $\mathrm{C}_{23} \mathrm{H}_{18} \mathrm{Cl}_{3} \mathrm{~N}_{4} \mathrm{O}_{2} \mathrm{~S}$ $[\mathrm{M}+\mathrm{H}]^{+}$519.0211, found 519.0211.

2-Chloro-5-(5-(6-chloro-3-(ethylthio)pyridin-2-yl)-1,2,4-oxadiazol-3-yl)-N-(2-fluorophenyl) benzamide (9h): White solid, yield $66.5 \%$, m.p. $213-217{ }^{\circ} \mathrm{C} ;{ }^{1} \mathrm{H}$ NMR $\left(500 \mathrm{MHz}\right.$, DMSO-d $\left.{ }_{6}\right)$ 
${ }^{1} \mathrm{H}$ NMR $(500 \mathrm{MHz}$, Chloroform- $d$ ) $\delta 10.56(\mathrm{~s}, 1 \mathrm{H}), 8.21(\mathrm{~m}, 2 \mathrm{H}), 8.14(\mathrm{~d}, J=8.5 \mathrm{~Hz}, 1 \mathrm{H})$, 7.91-7.82 (m, 2H), $7.80(\mathrm{~d}, J=8.5 \mathrm{~Hz}, 1 \mathrm{H}), 7.36-7.21(\mathrm{~m}, 3 \mathrm{H}), 3.16(\mathrm{q}, J=7.5 \mathrm{~Hz}, 2 \mathrm{H}), 1.31(\mathrm{t}$, $J=7.0 \mathrm{~Hz}, 3 \mathrm{H}) ;{ }^{13} \mathrm{C}$ NMR $\left(500 \mathrm{MHz}\right.$, DMSO- $\left.d_{6}\right){ }^{13} \mathrm{C}$ NMR $\left(126 \mathrm{MHz}\right.$, DMSO- $\left.d_{6}\right) \delta 172.42$, $164.54,158.63,145.88,143.26,139.54,138.66,138.23,137.37,136.91,132.59,131.18,129.68$, $127.80,127.49,125.78,125.19,124.60(\mathrm{~d}, J=10 \mathrm{~Hz}), 123.80,25.55,13.31$; HRMS calcd for $\mathrm{C}_{22} \mathrm{H}_{16} \mathrm{Cl}_{2} \mathrm{FN}_{4} \mathrm{O}_{2} \mathrm{~S}[\mathrm{M}+\mathrm{H}]^{+}$489.0350, found 489.0355 .

2-Chloro-5-(5-(6-chloro-3-(ethylthio)pyridin-2-yl)-1,2,4-oxadiazol-3-yl)-N-(4-fluorophenyl) benzamide (9i): Yellow solid, yield 61.6\%, m.p. $268-270{ }^{\circ} \mathrm{C} ;{ }^{1} \mathrm{H}$ NMR $\left(500 \mathrm{MHz}\right.$, DMSO- $\left.d_{6}\right)$ $\delta 10.76(\mathrm{~s}, 1 \mathrm{H}), 8.24-8.18(\mathrm{~m}, 2 \mathrm{H}), 8.13(\mathrm{~d}, J=9.0 \mathrm{~Hz}, 1 \mathrm{H}), 7.89-7.83(\mathrm{~m}, 1 \mathrm{H}), 7.82-7.72(\mathrm{~m}$, $3 \mathrm{H}), 7.22(\mathrm{t}, J=9.0 \mathrm{~Hz}, 2 \mathrm{H}), 3.16(\mathrm{q}, J=7.5 \mathrm{~Hz}, 2 \mathrm{H}), 1.30(\mathrm{t}, J=7.5 \mathrm{~Hz}, 3 \mathrm{H}) ;{ }^{13} \mathrm{C} \mathrm{NMR}$ (500 MHz, DMSO- $d_{6}$ ) $\delta 172.41,167.06,163.96,145.85,138.61,138.19,137.70,137.57,133.64$, 131.20, 129.64, 127.77, 127.28, 124.99, 121.74, 121.68, 115.67, 115.49, 25.50, 13.33; HRMS calcd for $\mathrm{C}_{22} \mathrm{H}_{16} \mathrm{Cl}_{2} \mathrm{FN}_{4} \mathrm{O}_{2} \mathrm{~S}[\mathrm{M}+\mathrm{H}]^{+} 489.0350$, found 489.0350 .

2-Chloro-5-(5-(6-chloro-3-(ethylthio)pyridin-2-yl)-1,2,4-oxadiazol-3-yl)-N-(2,6-difluorophenyl) benzamide (9j): Grey solid, yield $57.8 \%$, m.p. $258-261{ }^{\circ} \mathrm{C} ;{ }^{1} \mathrm{H}$ NMR $(500 \mathrm{MHz}, \mathrm{DMSO}-$ $\left.d_{6}\right) \delta 10.57(\mathrm{~s}, 1 \mathrm{H}), 8.27-8.18(\mathrm{~m}, 2 \mathrm{H}), 8.14(\mathrm{~d}, J=8.5 \mathrm{~Hz}, 1 \mathrm{H}), 7.90-7.76(\mathrm{~m}, 3 \mathrm{H}), 7.39$ $(\mathrm{t}, J=8.5 \mathrm{~Hz}, 1 \mathrm{H}), 7.16(\mathrm{t}, J=8.5 \mathrm{~Hz}, 1 \mathrm{H}), 3.16(\mathrm{q}, J=7.5 \mathrm{~Hz}, 2 \mathrm{H}), 1.31(\mathrm{t}, J=7.5 \mathrm{~Hz}, 3 \mathrm{H}) ;{ }^{13} \mathrm{C}$ NMR (500 MHz, DMSO- $d_{6}$ ) $\delta 172.46,167.78,167.11,158.28,152.27,146.15,145.92,138.69$, 138.25, 137.62, 137.22, 133.78, 131.25, 129.81, 127.84, 127.51, 117.00, 116.01, 111.58, 25.59, 13.33; HRMS calcd for $\mathrm{C}_{22} \mathrm{H}_{15} \mathrm{Cl}_{2} \mathrm{~F}_{2} \mathrm{~N}_{4} \mathrm{O}_{2} \mathrm{~S}[\mathrm{M}+\mathrm{H}]^{+}$507.0255, found 507.0258.

2-Chloro-5-(5-(6-chloro-3-(ethylthio)pyridin-2-yl)-1,2,4-oxadiazol-3-yl)-N-(2-chlorophenyl) benzamide (9k): White solid, yield $75.0 \%$, m.p. $207-208{ }^{\circ} \mathrm{C} ;{ }^{1} \mathrm{H}$ NMR $\left(500 \mathrm{MHz}\right.$, DMSO- $\left.d_{6}\right)$ $\delta 10.48(\mathrm{~s}, 1 \mathrm{H}), 8.30(\mathrm{~s}, 1 \mathrm{H}), 8.21(\mathrm{~d}, J=8.5 \mathrm{~Hz}, 1 \mathrm{H}), 8.14(\mathrm{~d}, J=8.5 \mathrm{~Hz}, 1 \mathrm{H}), 7.86(\mathrm{~d}$, $J=8.5 \mathrm{~Hz}, 1 \mathrm{H}), 7.80(\mathrm{~d}, J=8.5 \mathrm{~Hz}, 1 \mathrm{H}), 7.73(\mathrm{~d}, J=7.5 \mathrm{~Hz}, 1 \mathrm{H}), 7.58(\mathrm{~d}, J=8.0 \mathrm{~Hz}, 1 \mathrm{H})$, $7.42(\mathrm{t}, J=7.0 \mathrm{~Hz}, 1 \mathrm{H}), 7.32(\mathrm{t}, J=7.5 \mathrm{~Hz}, 1 \mathrm{H}), 3.16(\mathrm{q}, J=7.0 \mathrm{~Hz}, 2 \mathrm{H}), 1.32(\mathrm{t}, J=7.5 \mathrm{~Hz}$, $3 \mathrm{H}) ;{ }^{13} \mathrm{C}$ NMR $\left(500 \mathrm{MHz}\right.$, DMSO- $\left.d_{6}\right) \delta 172.45,167.07,164.37,145.87,140.12,138.25,137.55$, $137.44,133.65,133.32,131.24,130.76,129.93,129.85,127.78,127.29,125.06,119.40,119.32$, 118.33, 25.54, 13.35; HRMS calcd for $\mathrm{C}_{22} \mathrm{H}_{16} \mathrm{Cl}_{3} \mathrm{~N}_{4} \mathrm{O}_{2} \mathrm{~S}[\mathrm{M}+\mathrm{H}]^{+}$505.0054, found 505.0055.

2-Chloro-5-(5-(6-chloro-3-(ethylthio)pyridin-2-yl)-1,2,4-oxadiazol-3-yl)-N-(3-chlorophenyl) benzamide (9l): White solid, yield 75.4\%, m.p. $232-234{ }^{\circ} \mathrm{C} ;{ }^{1} \mathrm{H}$ NMR $\left(500 \mathrm{MHz}\right.$, DMSO- $d_{6}$ ) $\delta 10.90(\mathrm{~s}, 1 \mathrm{H}), 8.26-8.20(\mathrm{~m}, 2 \mathrm{H}), 8.14(\mathrm{~d}, J=8.5 \mathrm{~Hz}, 1 \mathrm{H}), 7.94(\mathrm{t}, J=1.5 \mathrm{~Hz}, 1 \mathrm{H}), 7.87$ $(\mathrm{d}, J=8.5 \mathrm{~Hz}, 1 \mathrm{H}), 7.80(\mathrm{~d}, J=9.0 \mathrm{~Hz}, 1 \mathrm{H}), 7.60(\mathrm{~d}, J=8.0 \mathrm{~Hz}, 1 \mathrm{H}), 7.42(\mathrm{t}$, $J=8.0 \mathrm{~Hz}, 1 \mathrm{H}), 7.24-7.18(\mathrm{~m}, 1 \mathrm{H}), 3.16(\mathrm{q}, J=7.0 \mathrm{~Hz}, 2 \mathrm{H}), 1.30(\mathrm{t}, J=7.0 \mathrm{~Hz}, 3 \mathrm{H}) ;{ }^{13} \mathrm{C} \mathrm{NMR}$ $\left(126 \mathrm{MHz}, \mathrm{DMSO}-d_{6}\right){ }^{13} \mathrm{C}$ NMR $(126 \mathrm{MHz}$, Chloroform- $d$ ) $\delta$ 177.49, 172.13, 169.39, 150.90, $145.27,143.38,142.55,138.73,138.41,136.35,136.28,135.80,134.93,132.82,132.51,130.17$, 129.06, 124.45, 124.38, 123.35, 30.60, 18.41; HRMS calcd for $\mathrm{C}_{22} \mathrm{H}_{16} \mathrm{Cl}_{3} \mathrm{~N}_{4} \mathrm{O}_{2} \mathrm{~S}[\mathrm{M}+\mathrm{H}]^{+}$ 505.0054 , found 505.0055 .

2-Chloro-5-(5-(6-chloro-3-(ethylthio)pyridin-2-yl)-1,2,4-oxadiazol-3-yl)-N-(4-chlorophenyl) benzamide (9m): White solid, yield 77.3\%, m.p. $242-243{ }^{\circ} \mathrm{C} ;{ }^{1} \mathrm{H}$ NMR $\left(500 \mathrm{MHz}\right.$, DMSO- $\left.d_{6}\right)$ $\delta 10.84(\mathrm{~s}, 1 \mathrm{H}), 8.24-8.18(\mathrm{~m}, 2 \mathrm{H}), 8.11(\mathrm{~d}, J=9.0 \mathrm{~Hz}, 1 \mathrm{H}), 7.85(\mathrm{~d}, J=8.0 \mathrm{~Hz}, 1 \mathrm{H}), 7.77(\mathrm{~m}$, $3 \mathrm{H}), 7.43(\mathrm{~d}, J=9.0 \mathrm{~Hz}, 2 \mathrm{H}), 3.14(\mathrm{q}, J=7.5 \mathrm{~Hz}, 2 \mathrm{H}), 1.29(\mathrm{t}, J=7.5 \mathrm{~Hz}, 3 \mathrm{H}) ;{ }^{13} \mathrm{C}$ NMR $\left(126 \mathrm{MHz}\right.$, DMSO- $\left.d_{6}\right) \delta 172.42,167.07,164.17,145.86,138.55,138.25,137.66,137.57,137.52$, $133.65,131.22,129.75,128.92,127.92,127.78,127.33,125.04,121.45,25.54,13.33$; HRMS calcd for $\mathrm{C}_{22} \mathrm{H}_{16} \mathrm{Cl}_{3} \mathrm{~N}_{4} \mathrm{O}_{2} \mathrm{~S}[\mathrm{M}+\mathrm{H}]^{+}$505.0054, found 505.0055.

$\mathrm{N}$-(4-Bromophenyl)-2-chloro-5-(5-(6-chloro-3-(ethylthio)pyridin-2-yl)-1,2,4-oxadiazol-3-yl) benzamide (9n): Yellow solid, yield 78.8\%, m.p. $267-269^{\circ} \mathrm{C} ;{ }^{1} \mathrm{H}$ NMR $\left(500 \mathrm{MHz}\right.$, DMSO- $\left.d_{6}\right)$ $\delta 10.84(\mathrm{~s}, 1 \mathrm{H}), 8.25-8.19(\mathrm{~m}, 2 \mathrm{H}), 8.13(\mathrm{~d}, J=8.5 \mathrm{~Hz}, 1 \mathrm{H}), 7.87(\mathrm{~d}, J=8.0 \mathrm{~Hz}, 1 \mathrm{H}), 7.79$ $(\mathrm{d}, J=9.0 \mathrm{~Hz}, 1 \mathrm{H}), 7.72(\mathrm{~d}, J=9 . \mathrm{Hz}, 2 \mathrm{H}), 7.57(\mathrm{~d}, J=8.5 \mathrm{~Hz}, 2 \mathrm{H}), 3.16(\mathrm{q}, J=7.5 \mathrm{~Hz}, 2 \mathrm{H})$, $1.30(\mathrm{t}, J=7.0 \mathrm{~Hz}, 3 \mathrm{H}) ;{ }^{13} \mathrm{C}$ NMR $\left(126 \mathrm{MHz}, \mathrm{DMSO}-d_{6}\right) \delta 172.43,167.08,164.20,145.88$, $138.60,138.24,138.08,137.57,133.64,131.84,131.24,129.77,127.80,127.33,125.04,121.83$, 115.99, 25.55, 13.35; HRMS calcd for $\mathrm{C}_{22} \mathrm{H}_{16} \mathrm{BrCl}_{2} \mathrm{~N}_{4} \mathrm{O}_{2} \mathrm{~S}[\mathrm{M}+\mathrm{H}]^{+} 548.9549$, found 548.9554 . 


\subsubsection{Synthesis of Target Compound $\mathbf{1 0}$}

To a stirred solution of compound $9(1.0 \mathrm{mmol})$ in DCM $(20 \mathrm{~mL})$, we added $\mathrm{mCPBA}$ $(0.5 \mathrm{~g}, 3.0 \mathrm{mmol})$. After stirring at room temperature for $3 \mathrm{~h}$, the mixture was poured into water and the $\mathrm{pH}$ was adjusted to 7-8 with $\mathrm{NaHCO}_{3}$. The organic layer was dried by $\mathrm{Na}_{2} \mathrm{SO}_{4}$ and removed under reduced pressure to give compound $\mathbf{1 0}$.

2-Chloro-5-(5-(6-chloro-3-(ethylsulfonyl)pyridin-2-yl)-1,2,4-oxadiazol-3-yl)-N-phenylbenzamide (10a): Pink solid, yield 83.7\%, m.p. $263-267^{\circ} \mathrm{C} ;{ }^{1} \mathrm{H}$ NMR (500 MHz, DMSO- $\left.d_{6}\right) \delta 10.70$ (s, $1 \mathrm{H}), 8.61(\mathrm{~d}, J=8.5 \mathrm{~Hz}, 1 \mathrm{H}), 8.23(\mathrm{~d}, J=2.5 \mathrm{~Hz}, 1 \mathrm{H}), 8.20(\mathrm{~m}, 2 \mathrm{H}), 7.85(\mathrm{~d}, J=8.5 \mathrm{~Hz}, 1 \mathrm{H})$, $7.74(\mathrm{~d}, J=8.0 \mathrm{~Hz}, 2 \mathrm{H}), 7.37(\mathrm{t}, J=8.0 \mathrm{~Hz}, 2 \mathrm{H}), 7.13(\mathrm{t}, J=7.5 \mathrm{~Hz}, 1 \mathrm{H}), 3.78(\mathrm{q}, J=7.5 \mathrm{~Hz}$, $2 \mathrm{H}), 1.26(\mathrm{t}, J=7.5 \mathrm{~Hz}, 3 \mathrm{H}) ;{ }^{13} \mathrm{C}$ NMR $\left(500 \mathrm{MHz}, \mathrm{DMSO}-d_{6}\right) \delta 171.62,166.81,163.79,154.63$, $142.95,142.22$, 138.64, 137.80, 135.62, 133.90, 131.16, 129.50, 128.82, 127.36, 124.27, 124.07, 119.71, 50.37, 6.64; HRMS calcd for $\mathrm{C}_{22} \mathrm{H}_{17} \mathrm{Cl}_{2} \mathrm{~N}_{4} \mathrm{O}_{4} \mathrm{~S}[\mathrm{M}+\mathrm{H}]^{+}$503.0342, found 503.0347.

2-Chloro-5-(5-(6-chloro-3-(ethylsulfonyl)pyridin-2-yl)-1,2,4-oxadiazol-3-yl)-N-(o-tolyl)benzamide (10b): White solid, yield 77.4\%, m.p. $257-259{ }^{\circ} \mathrm{C} ;{ }^{1} \mathrm{H}$ NMR (500 MHz, DMSO- $\left.d_{6}\right) \delta 10.18$ (s, $1 \mathrm{H}), 8.62(\mathrm{~d}, J=9.0 \mathrm{~Hz}, 1 \mathrm{H}), 8.26(\mathrm{~d}, J=2.0 \mathrm{~Hz}, 1 \mathrm{H}), 8.20(\mathrm{~d}, J=8.5 \mathrm{~Hz}, 2 \mathrm{H}), 7.85$ $(\mathrm{d}, J=8.5 \mathrm{~Hz}, 1 \mathrm{H}), 7.49(\mathrm{~d}, J=7.5 \mathrm{~Hz}, 1 \mathrm{H}), 7.31-7.20(\mathrm{~m}, 2 \mathrm{H}), 7.18(\mathrm{td}, J=7.5,1.5 \mathrm{~Hz}, 1 \mathrm{H})$, $3.79(\mathrm{q}, J=7.5 \mathrm{~Hz}, 2 \mathrm{H}), 2.31(\mathrm{~s}, 3 \mathrm{H}), 1.27(\mathrm{t}, J=7.5 \mathrm{~Hz}, 3 \mathrm{H}) ;{ }^{13} \mathrm{C}$ NMR $\left(500 \mathrm{MHz}\right.$, DMSO- $\left.d_{6}\right)$ $\delta 171.64,166.85,164.14,154.64,142.98,142.23,137.97,135.64,135.47,133.89,132.94,131.14$, 130.61, 130.44, 129.37, 128.79, 127.38, 126.07, 124.24, 50.37, 39.93, 17.90, 6.63; HRMS calcd for $\mathrm{C}_{23} \mathrm{H}_{19} \mathrm{Cl}_{2} \mathrm{~N}_{4} \mathrm{O}_{4} \mathrm{~S}[\mathrm{M}+\mathrm{H}]^{+} 517.0499$, found 517.0500.

2-Chloro-5-(5-(6-chloro-3-(ethylsulfonyl)pyridin-2-yl)-1,2,4-oxadiazol-3-yl)-N-(p-tolyl)benzamide (10c): White solid, yield $84.3 \%$, m.p. $260-261{ }^{\circ} \mathrm{C} ;{ }^{1} \mathrm{H}$ NMR $\left(500 \mathrm{MHz}\right.$, DMSO- $\left.d_{6}\right) \delta$ $10.59(\mathrm{~s}, 1 \mathrm{H}), 8.61(\mathrm{~d}, J=8.5 \mathrm{~Hz}, 1 \mathrm{H}), 8.26-8.15(\mathrm{~m}, 3 \mathrm{H}), 7.84(\mathrm{~d}, J=8.5 \mathrm{~Hz}, 1 \mathrm{H}), 7.61$ $(\mathrm{d}, J=8.5 \mathrm{~Hz}, 2 \mathrm{H}), 7.17(\mathrm{~d}, J=8.0 \mathrm{~Hz}, 2 \mathrm{H}), 3.77(\mathrm{q}, J=7.5 \mathrm{~Hz}, 2 \mathrm{H}), 2.28(\mathrm{~s}, 3 \mathrm{H}), 1.26$ $(\mathrm{t}, J=7.0 \mathrm{~Hz}, 3 \mathrm{H}) ;{ }^{13} \mathrm{C}$ NMR $\left(500 \mathrm{MHz}\right.$, DMSO- $\left.d_{6}\right){ }^{13} \mathrm{C}$ NMR $\left(126 \mathrm{MHz}, \mathrm{DMSO}-d_{6}\right) \delta 171.61$, 166.81, 163.60, 154.63, 142.94, 142.20, 137.85, 136.11, 135.60, 133.90, 133.14, 131.16, 129.45, $129.18,128.78,127.32,124.22,119.72,50.36,20.46,6.61$; HRMS calcd for $\mathrm{C}_{23} \mathrm{H}_{19} \mathrm{Cl}_{2} \mathrm{~N}_{4} \mathrm{O}_{4} \mathrm{~S}$ $[\mathrm{M}+\mathrm{H}]^{+}$517.0499, found 517.0505.

$\mathrm{N}$-(4-(tert-Butyl)phenyl)-2-chloro-5-(5-(6-chloro-3-(ethylsulfonyl)pyridin-2-yl)-1,2,4-oxadiazol3-yl)benzamide (10d): Grey yield, yield 79.2\%, m.p. $268-270{ }^{\circ} \mathrm{C} ;{ }^{1} \mathrm{H}$ NMR $(500 \mathrm{MHz}, \mathrm{DMSO}$ $\left.d_{6}\right) \delta 10.61(\mathrm{~s}, 1 \mathrm{H}), 8.62(\mathrm{~d}, J=8.5 \mathrm{~Hz}, 1 \mathrm{H}), 8.24-8.12(\mathrm{~m}, 3 \mathrm{H}), 7.85(\mathrm{~d}, J=9.0 \mathrm{~Hz}, 1 \mathrm{H}), 7.64$ $(\mathrm{d}, J=8.5 \mathrm{~Hz}, 2 \mathrm{H}), 7.39(\mathrm{~d}, J=8.5 \mathrm{~Hz}, 2 \mathrm{H}), 3.78(\mathrm{q}, J=7.5 \mathrm{~Hz}, 2 \mathrm{H}), 1.33-1.27(\mathrm{~m}, 12 \mathrm{H}) ;{ }^{13} \mathrm{C}$ NMR (500 MHz, DMSO- $d_{6}$ ) $\delta 176.85,172.05,168.89,159.88,151.78,148.21,147.45,143.08$, $141.26,140.83$, 139.14, 136.39, 134.64, 134.06, 132.50, 130.66, 129.46, 124.77, 55.61, 39.28, 36.36, 11.87; HRMS calcd for $\mathrm{C}_{26} \mathrm{H}_{25} \mathrm{Cl}_{2} \mathrm{~N}_{4} \mathrm{O}_{4} \mathrm{~S}[\mathrm{M}+\mathrm{H}]^{+} 559.0968$, found 559.0971.

2-Chloro-5-(5-(6-chloro-3-(ethylsulfonyl)pyridin-2-yl)-1,2,4-oxadiazol-3-yl)-N-(2,4-dimethylphenyl) benzamide (10e): White solid, yield 78.4\%,. m.p. $246-248{ }^{\circ} \mathrm{C} ;{ }^{1} \mathrm{H}$ NMR $\left(500 \mathrm{MHz}, \mathrm{DMSO}-d_{6}\right)$ $\delta 10.09(\mathrm{~s}, 1 \mathrm{H}), 8.61(\mathrm{~d}, J=8.5 \mathrm{~Hz}, 1 \mathrm{H}), 8.24(\mathrm{~d}, J=2.0 \mathrm{~Hz}, 1 \mathrm{H}), 8.22-8.17(\mathrm{~m}, 2 \mathrm{H}), 7.84$ $(\mathrm{d}, J=8.5 \mathrm{~Hz}, 1 \mathrm{H}), 7.34(\mathrm{~d}, J=8.0 \mathrm{~Hz}, 1 \mathrm{H}), 7.08(\mathrm{~s}, 1 \mathrm{H}), 7.04(\mathrm{dd}, J=8.0,2.0 \mathrm{~Hz}, 1 \mathrm{H}), 3.79$ $(\mathrm{q}, J=7.0 \mathrm{~Hz}, 2 \mathrm{H}), 2.27(\mathrm{~d}, J=7.5 \mathrm{~Hz}, 6 \mathrm{H}), 1.27(\mathrm{t}, J=7.5 \mathrm{~Hz}, 3 \mathrm{H}) ;{ }^{13} \mathrm{C}$ NMR $(500 \mathrm{MHz}$, DMSO- $\left.d_{6}\right) \delta 171.62,166.84,164.16,154.64,142.96,142.21,138.02,135.62,135.34,133.87$, $132.84,132.81,131.12,130.92,129.32,128.78,127.34,126.57,125.93,124.20,50.37,20.49,17.81$, 6.62; HRMS calcd for $\mathrm{C}_{24} \mathrm{H}_{21} \mathrm{Cl}_{2} \mathrm{~N}_{4} \mathrm{O}_{4} \mathrm{~S}[\mathrm{M}+\mathrm{H}]^{+} 531.0655$, found 531.0660.

2-Chloro-5-(5-(6-chloro-3-(ethylsulfonyl)pyridin-2-yl)-1,2,4-oxadiazol-3-yl)-N-(3-(trifluoromethyl) phenyl)benzamide (10f): Yellow solid, yield 76.8\%, m.p. $214-217^{\circ} \mathrm{C}$; H NMR $(500 \mathrm{MHz}$, DMSO- $\left.d_{6}\right) \delta 11.07(\mathrm{~s}, 1 \mathrm{H}), 8.61(\mathrm{~d}, J=8.5 \mathrm{~Hz}, 1 \mathrm{H}), 8.30(\mathrm{~d}, J=2.0 \mathrm{~Hz}, 1 \mathrm{H}), 8.26-8.17(\mathrm{~m}$, $3 \mathrm{H}), 7.92(\mathrm{~d}, J=8.5 \mathrm{~Hz}, 1 \mathrm{H}), 7.87(\mathrm{~d}, J=8.5 \mathrm{~Hz}, 1 \mathrm{H}), 7.62(\mathrm{t}, J=8.0 \mathrm{~Hz}, 1 \mathrm{H}), 7.50(\mathrm{~d}$, $J=7.5 \mathrm{~Hz}, 1 \mathrm{H}), 3.78(\mathrm{q}, J=7.5 \mathrm{~Hz}, 2 \mathrm{H}), 1.26(\mathrm{t}, J=7.0 \mathrm{~Hz}, 3 \mathrm{H}) ;{ }^{13} \mathrm{C}$ NMR $(500 \mathrm{MHz}$, DMSO- $d$ 6) $\delta 171.64,166.77,164.23,154.62,142.95,142.20,139.37,137.23,135.61,133.90,131.23,130.13$, $129.80,129.54(\mathrm{~d}, J=32.1 \mathrm{~Hz}), 128.79,127.49,125.10,124.32,123.30,120.44$ (d), 115.78 $(\mathrm{d}, J=4.0 \mathrm{~Hz}), 50.37,6.62$; HRMS calcd for $\mathrm{C}_{23} \mathrm{H}_{16} \mathrm{Cl}_{2} \mathrm{~F}_{3} \mathrm{~N}_{4} \mathrm{O}_{4} \mathrm{~S}[\mathrm{M}+\mathrm{H}]^{+}$571.0216, found 571.0222.

2-Chloro-N-(3-chloro-2-methylphenyl)-5-(5-(6-chloro-3-(ethylsulfonyl)pyridin-2-yl)-1,2,4oxadiazol-3-yl)benzamide (10g): Yellow solid, yield 69.1\%, m.p. $255-256{ }^{\circ} \mathrm{C} ;{ }^{1} \mathrm{H}$ NMR 
$\left(500 \mathrm{MHz}, \mathrm{DMSO}-\mathrm{d}_{6}\right) \delta 10.44(\mathrm{~s}, 1 \mathrm{H}), 8.62(\mathrm{~d}, J=8.5 \mathrm{~Hz}, 1 \mathrm{H}), 8.29(\mathrm{~d}, J=2.0 \mathrm{~Hz}, 2 \mathrm{H})$, $8.21(\mathrm{~m}, 1 \mathrm{H}), 7.86(\mathrm{~d}, J=8.5 \mathrm{~Hz}, 1 \mathrm{H}), 7.46(\mathrm{~d}, J=8.0 \mathrm{~Hz}, 1 \mathrm{H}), 7.38(\mathrm{~d}, J=7.5 \mathrm{~Hz}, 1 \mathrm{H})$, $7.28(\mathrm{t}, J=8.0 \mathrm{~Hz}, 1 \mathrm{H}), 3.79(\mathrm{q}, J=7.0 \mathrm{~Hz}, 2 \mathrm{H}), 2.34(\mathrm{~s}, 3 \mathrm{H}), 1.27(\mathrm{t}, J=7.5 \mathrm{~Hz}, 3 \mathrm{H}) ;{ }^{13} \mathrm{C}$ NMR (500 MHz, DMSO- $\left.d_{6}\right){ }^{13} \mathrm{C}$ NMR $\left(126 \mathrm{MHz}, \mathrm{DMSO}-d_{6}\right) \delta 171.64,166.81,164.30,154.63$, $142.97,142.21,137.60,137.00,135.63,133.88,131.45,131.17,129.55,128.81,128.78,127.40$, $127.01,126.95,125.34,124.28,50.36,15.19,6.64$; HRMS calcd for $\mathrm{C}_{23} \mathrm{H}_{18} \mathrm{Cl}_{3} \mathrm{~N}_{4} \mathrm{O}_{4} \mathrm{~S}[\mathrm{M}+\mathrm{H}]^{+}$ 551.0109 , found 551.0108 .

2-Chloro-5-(5-(6-chloro-3-(ethylsulfonyl)pyridin-2-yl)-1,2,4-oxadiazol-3-yl)-N-(2-fluorophenyl) benzamide (10h): Brown solid, yield 73.7\%, m.p. $253-257{ }^{\circ} \mathrm{C} ;{ }^{1} \mathrm{H}$ NMR (500 MHz, DMSO-d 6 ) $\delta 10.58(\mathrm{~s}, 1 \mathrm{H}), 8.61(\mathrm{~d}, J=8.5 \mathrm{~Hz}, 1 \mathrm{H}), 8.27-8.11(\mathrm{~m}, 3 \mathrm{H}), 7.94-7.81(\mathrm{~m}, 2 \mathrm{H}), 7.38-7.17$ $(\mathrm{m}, 3 \mathrm{H}), 3.78(\mathrm{q}, J=7.0 \mathrm{~Hz}, 2 \mathrm{H}), 1.26(\mathrm{t}, J=7.0 \mathrm{~Hz}, 3 \mathrm{H}) ;{ }^{13} \mathrm{C}$ NMR $\left(500 \mathrm{MHz}, \mathrm{DMSO}-d_{6}\right)$ ${ }^{13} \mathrm{C}$ NMR $\left(126 \mathrm{MHz}, \mathrm{DMSO}-d_{6}\right) \delta 171.62,166.80,166.07,154.64,142.94,142.19,137.26$, $135.60,133.97,133.31,132.69,131.16,130.64,129.60,128.77,127.87,127.51,125.62,124.45$, $115.80(\mathrm{~d}, \mathrm{~J}=77.5 \mathrm{~Hz}), 50.37,6.58$; HRMS calcd for $\mathrm{C}_{22} \mathrm{H}_{16} \mathrm{Cl}_{2} \mathrm{FN}_{4} \mathrm{O}_{4} \mathrm{~S}[\mathrm{M}+\mathrm{H}]^{+} 521.0248$, found 521.0255 .

2-Chloro-5-(5-(6-chloro-3-(ethylsulfonyl)pyridin-2-yl)-1,2,4-oxadiazol-3-yl)-N-(2-fluorophenyl) benzamide (10i): Yellow solid, yield 71.6\%, m.p. $229-231{ }^{\circ} \mathrm{C} ;{ }^{1} \mathrm{H}$ NMR (500 MHz, DMSO-d $\left.d_{6}\right)$ $\delta 10.75(\mathrm{~s}, 1 \mathrm{H}), 8.61(\mathrm{~d}, J=8.5 \mathrm{~Hz}, 1 \mathrm{H}), 8.24(\mathrm{~d}, J=2.0 \mathrm{~Hz}, 1 \mathrm{H}), 8.23-8.18(\mathrm{~m}, 2 \mathrm{H}), 7.85$ $(\mathrm{d}, J=8.5 \mathrm{~Hz}, 1 \mathrm{H}), 7.78-7.71(\mathrm{~m}, 2 \mathrm{H}), 7.22(\mathrm{t}, J=9.0 \mathrm{~Hz}, 2 \mathrm{H}), 3.78(\mathrm{q}, J=7.0 \mathrm{~Hz}, 2 \mathrm{H})$, $1.26(\mathrm{t}, J=7.5 \mathrm{~Hz}, 3 \mathrm{H}) ;{ }^{13} \mathrm{C}$ NMR $\left(500 \mathrm{MHz}, \mathrm{DMSO}-d_{6}\right){ }^{13} \mathrm{C}$ NMR $\left(126 \mathrm{MHz}, \mathrm{DMSO}-d_{6}\right) \delta$ $171.62,166.79,163.71,157.51,154.63,142.94,142.20,137.61,135.60,134.97,133.90,131.18$, $129.58,128.78,127.37,124.27,121.60,115.43(\mathrm{~d}, J=88.0 \mathrm{~Hz}), 50.37,6.62$; HRMS calcd for $\mathrm{C}_{22} \mathrm{H}_{16} \mathrm{Cl}_{2} \mathrm{FN}_{4} \mathrm{O}_{4} \mathrm{~S}[\mathrm{M}+\mathrm{H}]^{+}$521.0248, found 521.0251 .

2-Chloro-5-(5-(6-chloro-3-(ethylsulfonyl)pyridin-2-yl)-1,2,4-oxadiazol-3-yl)-N-(2,6-difluorophenyl) benzamide (10j): Brown solid, yield 72.8\%, m.p. 237-239 ${ }^{\circ} \mathrm{C} ;{ }^{1} \mathrm{H}$ NMR (500 MHz, DMSO- $\left.d_{6}\right)$ $\delta 10.56(\mathrm{~s}, 1 \mathrm{H}), 8.61(\mathrm{~d}, J=8.5 \mathrm{~Hz}, 1 \mathrm{H}), 8.25(\mathrm{~m}, 1 \mathrm{H}), 8.21(\mathrm{~d}, J=8.5 \mathrm{~Hz}, 2 \mathrm{H}), 7.89-7.82(\mathrm{~m}$, $2 \mathrm{H}), 7.42-7.34(\mathrm{~m}, 1 \mathrm{H}), 7.19-7.12(\mathrm{~m}, 1 \mathrm{H}), 3.78(\mathrm{q}, J=7.5 \mathrm{~Hz}, 2 \mathrm{H}), 1.27(\mathrm{t}, J=7.5 \mathrm{~Hz}, 3 \mathrm{H})$; ${ }^{13} \mathrm{C}$ NMR $\left(500 \mathrm{MHz}, \mathrm{DMSO}-d_{6}\right) \delta 171.63,166.79,164.35,154.64,142.94,142.18,137.08,135.61$, 133.97, 131.19, 129.68, 128.79, 127.52, 127.17 (d, $J=7.5 \mathrm{~Hz}), 124.17,111.40$ (d, $J=12.5 \mathrm{~Hz})$, $111.22,104.60,104.40(\mathrm{~d}, J=9.0 \mathrm{~Hz}), 104.19,50.36,6.58$; HRMS calcd for $\mathrm{C}_{22} \mathrm{H}_{15} \mathrm{Cl}_{2} \mathrm{~F}_{2} \mathrm{~N}_{4} \mathrm{O}_{4} \mathrm{~S}$ $[\mathrm{M}+\mathrm{H}]^{+} 539.0154$, found 539.0159 .

2-Chloro-5-(5-(6-chloro-3-(ethylsulfonyl)pyridin-2-yl)-1,2,4-oxadiazol-3-yl)-N-(2-chlorophenyl) benzamide (10k): White solid, yield 82.0\%, m.p. 209-212 ${ }^{\circ} \mathrm{C} ;{ }^{1} \mathrm{H}$ NMR $(500 \mathrm{MHz}, \mathrm{DMSO}-$ $\left.d_{6}\right) \delta 10.50(\mathrm{~s}, 1 \mathrm{H}), 8.62(\mathrm{~d}, J=8.0 \mathrm{~Hz}, 1 \mathrm{H}), 8.34-8.14(\mathrm{~m}, 3 \mathrm{H}), 7.93-7.82(\mathrm{~m}, 1 \mathrm{H}), 7.73$ $(\mathrm{d}, J=7.0 \mathrm{~Hz}, 1 \mathrm{H}), 7.58(\mathrm{~d}, J=7.5 \mathrm{~Hz}, 1 \mathrm{H}), 7.48-7.38(\mathrm{~m}, 1 \mathrm{H}), 7.36-7.29(\mathrm{~m}, 1 \mathrm{H}), 3.86-3.73$ $(\mathrm{m}, 2 \mathrm{H}), 1.28(\mathrm{t}, J=7.0 \mathrm{~Hz}, 3 \mathrm{H}) ;{ }^{13} \mathrm{C}$ NMR $\left(500 \mathrm{MHz}, \mathrm{DMSO}-d_{6}\right){ }^{13} \mathrm{C} \mathrm{NMR}(126 \mathrm{MHz}$, DMSO-d $\left.d_{6}\right) \delta 171.62,166.79,164.38,154.66,142.94,142.18,135.58,134.07,134.00,131.23$, $129.68,129.63,128.79,128.77,127.87,127.84,127.73,127.59,127.48,124.15,50.38,6.61$; HRMS calcd for $\mathrm{C}_{22} \mathrm{H}_{16} \mathrm{Cl}_{3} \mathrm{~N}_{4} \mathrm{O}_{4} \mathrm{~S}[\mathrm{M}+\mathrm{H}]^{+} 536.9952$, found 536.9958 .

2-Chloro-5-(5-(6-chloro-3-(ethylsulfonyl)pyridin-2-yl)-1,2,4-oxadiazol-3-yl)-N-(2-chlorophenyl) benzamide (10l): Yellow solid, yield 85.4\%, m.p. 217-218 ${ }^{\circ} \mathrm{C} ;{ }^{1} \mathrm{H}$ NMR (500 MHz, DMSO$\left.d_{6}\right) \delta 10.91(\mathrm{~s}, 1 \mathrm{H}), 8.62(\mathrm{~d}, J=8.5 \mathrm{~Hz}, 1 \mathrm{H}), 8.31-8.17(\mathrm{~m}, 3 \mathrm{H}), 7.97-7.84(\mathrm{~m}, 2 \mathrm{H}), 7.60(\mathrm{~d}$, $J=7.5 \mathrm{~Hz}, 1 \mathrm{H}), 7.42(\mathrm{t}, J=8.0 \mathrm{~Hz}, 1 \mathrm{H}), 7.21(\mathrm{~d}, J=7.5 \mathrm{~Hz}, 1 \mathrm{H}), 3.79(\mathrm{q}, J=7.0 \mathrm{~Hz}, 2 \mathrm{H})$, $1.26(\mathrm{t}, J=7.0 \mathrm{~Hz}, 3 \mathrm{H}) ;{ }^{13} \mathrm{C}$ NMR $\left(126 \mathrm{MHz}, \mathrm{DMSO}-d_{6}\right){ }^{13} \mathrm{C}$ NMR $\left(126 \mathrm{MHz}, \mathrm{DMSO}-d_{6}\right)$ $\delta 171.63,166.77,164.09,154.64,142.93,142.18,139.97,137.32,135.58,133.87,133.15,131.23$, $130.58,129.75,128.79,127.40,124.30,123.85,119.19,118.15,50.37,6.62$; HRMS calcd for $\mathrm{C}_{22} \mathrm{H}_{16} \mathrm{Cl}_{3} \mathrm{~N}_{4} \mathrm{O}_{4} \mathrm{~S}[\mathrm{M}+\mathrm{H}]^{+}$536.9952, found 536.9959 .

2-Chloro-5-(5-(6-chloro-3-(ethylsulfonyl)pyridin-2-yl)-1,2,4-oxadiazol-3-yl)-N-(2-chlorophenyl) benzamide (10m): White solid, yield 84.3\%, m.p. $233-236{ }^{\circ} \mathrm{C} ;{ }^{1} \mathrm{H}$ NMR (500 MHz, DMSO- $\left.d_{6}\right)$ ${ }^{13} \mathrm{C}$ NMR (126 MHz, DMSO-d 6 ) $\delta 171.63,166.77,164.09,154.64,142.93,142.18,139.97$, $137.32,135.58,133.87,133.15,131.23,130.58,129.75,128.79,127.40,124.30,123.85,119.19$, 118.15, 50.37, 6.62; ${ }^{13} \mathrm{C}$ NMR (126 MHz, DMSO- $\left.d_{6}\right){ }^{13} \mathrm{C}$ NMR $\left(126 \mathrm{MHz}, \mathrm{DMSO}-d_{6}\right) \delta 171.62$, $166.78,163.88,154.64,142.94,142.19,137.53,137.47,135.59,133.88,131.20,129.66,128.78$, 
$128.75,127.74,127.39,124.28,121.29,50.37,6.62 ; \mathrm{HRMS}$ calcd for $\mathrm{C}_{22} \mathrm{H}_{16} \mathrm{Cl}_{3} \mathrm{~N}_{4} \mathrm{O}_{4} \mathrm{~S}[\mathrm{M}+\mathrm{H}]^{+}$ 536.9952 , found 521.0251 .

$\mathrm{N}$-(4-Bromophenyl)-2-chloro-5-(5-(6-chloro-3-(ethylsulfonyl)pyridin-2-yl)-1,2,4-oxadiazol-3yl)benzamide (10n): Yellow solid, yield 78.3\%, m.p. $263-265^{\circ} \mathrm{C} ;{ }^{1} \mathrm{H}$ NMR $(500 \mathrm{MHz}, \mathrm{DMSO}-$ $\left.d_{6}\right) \delta 10.83(\mathrm{~s}, 1 \mathrm{H}), 8.61(\mathrm{~d}, J=8.5 \mathrm{~Hz}, 1 \mathrm{H}), 8.33-8.11(\mathrm{~m}, 3 \mathrm{H}), 7.85(\mathrm{~d}, J=8.0 \mathrm{~Hz}, 1 \mathrm{H}), 7.71$ $(\mathrm{d}, J=8.5 \mathrm{~Hz}, 2 \mathrm{H}), 7.56(\mathrm{~d}, J=8.5 \mathrm{~Hz}, 2 \mathrm{H}), 3.77(\mathrm{q}, J=7.5 \mathrm{~Hz}, 2 \mathrm{H}), 1.26(\mathrm{t}, J=7.5 \mathrm{~Hz}, 3 \mathrm{H})$; ${ }^{13} \mathrm{C}$ NMR $\left(126 \mathrm{MHz}\right.$, DMSO- $\left.d_{6}\right){ }^{13} \mathrm{C}$ NMR $\left(126 \mathrm{MHz}\right.$, DMSO- $\left.d_{6}\right) \delta 171.61,166.77,163.91$, $154.65,142.93,142.18,137.93,137.44,135.58,133.87,131.66,131.21,129.67,128.78,127.38$, 124.27, 121.67, 115.81, 50.38, 6.61; HRMS calcd for $\mathrm{C}_{22} \mathrm{H}_{16} \mathrm{BrCl}_{2} \mathrm{~N}_{4} \mathrm{O}_{4} \mathrm{~S}[\mathrm{M}+\mathrm{H}]^{+}$580.9447, found 580.9449 .

\subsection{Biological Activity and Toxicity Determination}

The insecticidal and fungicidal activities were investigated in the National Pesticide Engineering Research Centre, Nankai University, according to references [49,50], and the results of the activity test are shown in Table 1.

Through acute exposure, we assessed the toxicity of compounds $9 \mathrm{f}$ and $\mathbf{1 0 f}$ on zebrafish embryo. According to the preliminary exposure experiments, a series of gradient concentrations of compounds $9 \mathrm{f}$ and $\mathbf{1 0 f}$ were set on the basis of mortality rates in the range of $10-95 \%$. $\mathrm{LC}_{50}$ values for zebrafish embryos exposed to compound $9 \mathrm{f}$ or $10 \mathrm{f}$ from 6 to $96 \mathrm{hpf}$ : control (0 mg/L of 9f), 1, 5, $10 \mathrm{mg} / \mathrm{L}$ of $9 f$; control (0 mg/L of 10f), 5, $10 \mathrm{mg} / \mathrm{L}$ of 10f. The $\mathrm{LC}_{50}$ (median lethal concentration) values were computed by the Boltzmann equation [51,52]. The observational indexes included $96 \mathrm{hpf}$ mortality rate, $72 \mathrm{hpf}$ hatching rate, and $96 \mathrm{hpf}$ malformation rate.

\section{Conclusions}

In conclusion, a series of novel benzamides containing 1,2,4-oxadiazole moiety were designed by bioisosterism and were synthesized easily via thioetherification, cyclization, aminolysis, and oxidation reactions. Their structures were confirmed by ${ }^{1} \mathrm{H} N M R,{ }^{13} \mathrm{C}$ NMR, and HRMS. The bioassay results showed that some of the title compounds displayed excellent fungicidal activities against Botrytis cinereal at $100 \mathrm{mg} / \mathrm{L}$. For example, 10a (84.4\%), 10d $(83.6 \%), 10 e(83.3 \%), 10 f(83.1 \%), 10 \mathrm{i}(83.3 \%)$, and $101(83.6 \%)$ were better than the control fungicide pyraclostrobin (81.4\%). In addition, the acute toxicity of $\mathbf{1 0 f}$ to zebrafish embryo was $20.58 \mathrm{mg} / \mathrm{L}$, which was classified as a low toxicity compound. Therefore, these compounds could potentially be the lead compounds for further study.

Supplementary Materials: The following are available online at https:/ / www.mdpi.com/1422-006 $7 / 22 / 5 / 2367 /$ s1.

Author Contributions: S.Y., X.-Y.T., T.-Y.M., L.D., C.-L.R., and W.-Q.Z. carried out experimental work; S.Y. prepared the manuscript; C.-X.T. designed the material and supervised the project; and X.-H.L. and C.-X.T. revised the paper. All authors have read and agreed to the published version of the manuscript.

Funding: This work was funded by the Wynca Group and Siga Co. Ltd. R\&D Program, grant numbers KYY-HX-20180412 and KYY-HX-20180737.

Data Availability Statement: Samples of the compounds are not available from the authors.

Acknowledgments: We acknowledge Hui-Li Wang for support with the toxicity determination.

Conflicts of Interest: The authors declare no conflict of interest.

\section{Abbreviations}

HRMS (High Resolution Mass Spectrometer), DMF (N,N-Dimethylformamide), mCPBA (3-Chloroperbenzoic acid), DMS (Dimethyl sulfate), rt (Room temperature), KTB (Potassium t-butoxide), THF (Tetrahydrofuran), EtOAc (Ethyl acetate), EDCI (1-Ethyl-3-(3-dimethylaminopropyl)carbodiimide hydrochloride), SAR (Structure activity relationships). 


\section{References}

1. Verger, P.J.P.; Boobis, A.R. Reevaluate pesticides for food security and safety. Science 2013, 341, 717-718. [CrossRef]

2. Wang, B.L.; Zhu, H.W.; Ma, Y.; Xiong, L.X.; Li, Y.Q.; Zhao, Y.; Zhang, J.F.; Chen, Y.W.; Zhou, S.; Li, Z.M. Synthesis, insecticidal activities, and SAR studies of novel pyridylpyrazole acid derivatives based on amide bridge modification of anthranilic diamide insecticides. J. Agric. Food Chem. 2013, 61, 5483-5493. [CrossRef] [PubMed]

3. Oerke, E.C. Crop losses to pests. J. Agric. Sci. 2006, 144, 31-43. [CrossRef]

4. Shi:, J.J.; Ren, G.H.; Wu, N.-J.; Weng, J.-Q.; Xu, T.-M.; Liu, X.-H.; Tan, C.-X. Design, synthesis and insecticidal activities of novel anthranilic diamides containing polyfluoroalkyl pyrazole moiety. Chin. Chem. Lett. 2017, 28, 1727-1730. [CrossRef]

5. Alnufaie, R.; Alsup, N.; Whitt, J.; Chambers, A.S.; Gilmore, D.; Alam, M.A. Synthesis and Antimicrobial Studies of CoumarinSubstituted Pyrazole Derivatives as Potent Anti-Staphylococcus aureus Agents. Molecules 2020, 25, 2758. [CrossRef] [PubMed]

6. Avenot, H.F.; Michailides, T.J. Progress in understanding molecular mechanisms and evolution of resistance to succinate dehydrogenase inhibiting (SDHI) fungicides in phytopathogenic fungi. Crop Prot. 2010, 29, 643-651. [CrossRef]

7. Fuentes-Gutiérrez, A.; Curiel-Quesada, E.; Correa-Basurto, J.; Martínez-Muñoz, A.; Reyes-Arellano, A. N-Heterocycles Scaffolds as Quorum Sensing Inhibitors. Design, Synthesis, Biological and Docking Studies. Int. J. Mol. Sci. 2020, $21,9512$.

8. Hu, Y.; Li, C.Y.; Wang, X.M.; Yang, Y.H.; Zhu, H.L. 1,3,4-Thiadiazole: Synthesis, reactions, and applications in medicinal, agricultural, and materials chemistry. Chem. Rev. 2014, 114, 5572-5610. [CrossRef] [PubMed]

9. Guo, X.; Zhao, B.; Fan, Z.J.; Yang, D.Y.; Zhang, N.L.; Wu, Q.F.; Yu, B.; Zhou, S.; Kalinina, T.A.; Belskaya, N.P. Discovery of novel thiazole carboxamides as antifungal succinate dehydrogenase inhibitors. J. Agric. Food Chem. 2019, 67, 1647-1655. [CrossRef]

10. Duchowicz, P.R.; Vitale, M.G.; Castro, E.A.; Fernández, M.; Caballero, J. QSAR analysis for heterocyclic antifungals. Bioorg. Med. Chem. 2007, 15, 2680-2689. [CrossRef]

11. Du, S.J.; Lu, H.Z.; Yang, D.Y.; Li, H.; Gu, X.L.; Wan, C.; Jia, C.M.; Wang, M.; Li, X.Y.; Qin, Z.H. Synthesis, antifungal activity and QSAR of some novel carboxylic acid amides. Molecules 2015, 20, 4071-4087. [CrossRef]

12. Hou, Y.P.; Mao, X.W.; Wang, J.X.; Zhan, S.W.; Zhou, M.G. Sensitivity of Fusarium asiaticum to a novel succinate dehydrogenase inhibitor fungicide pydiflumetofen. Crop Prot. 2017, 96, 237-244. [CrossRef]

13. Liu, X.H.; Qiao, L.; Zhai, Z.W.; Cai, P.P.; Cantrell, C.L.; Tan, C.X.; Weng, J.Q.; Han, L.; Wu, H.K. Novel 4-Pyrazole Carboxamide Derivatives Containing Flexible Chain Motif: Design, Synthesis and Antifungal Activity. Pest Manag. Sci. 2019, 75, 2892-2900. [CrossRef]

14. Wu, Y.Y.; Shao, W.B.; Zhu, J.J.; Long, Z.Q.; Liu, L.W.; Wang, P.Y.; Li, Z.; Yang, S. Novel 1, 3, 4-Oxadiazole-2-carbohydrazides as Prospective Agricultural Antifungal Agents Potentially Targeting Succinate Dehydrogenase. J. Agric. Food Chem. 2019, 67, 13892-13903. [CrossRef]

15. Hua, X.W.; Liu, W.R.; Su, Y.Y.; Liu, X.H.; Liu, J.B.; Liu, N.N.; Wang, G.Q.; Jiao, X.Q.; Fan, X.Y.; Xue, C.M.; et al. Studies on the novel pyridine sulfide containing SDH based heterocyclic amide fungicide. Pest Manag. Sci. 2020, 76, 2368-2378. [CrossRef]

16. Eckroat, T.J.; Manross, D.L.; Cowan, S.C. Merged Tacrine-Based, Multitarget-Directed Acetylcholinesterase Inhibitors 2015-Present: Synthesis and Biological Activity. Int. J. Mol. Sci. 2020, 21, 5696. [CrossRef]

17. Zhang, A.G.; Zhou, J.Y.; Tao, K.; Hou, T.P.; Jin, H. Design, synthesis and antifungal evaluation of novel pyrazole carboxamides with diarylamines scaffold as potent succinate dehydrogenase inhibitors. Bioorg. Med. Chem. Lett. 2018, 28, 3042-3045. [CrossRef] [PubMed]

18. Wang, L.; Dai, F.Y.; Zhu, J.; Dong, K.K.; Wang, Y.L.; Chen, T. Synthesis and antibacterial activities of pleuromutilin derivatives with thiazole-5-carboxamide and thioether moiety. J. Chem. Res. 2011, 35, 313-316. [CrossRef]

19. Fu, Q.; Cai, P.P.; Cheng, L.; Zhong, L.K.; Shen, Z.H.; Han, L.; Liu, X.H. Synthesis and herbicidal activity of novel pyrazole aromatic ketone analogs as HPPD inhibitor. Pest Manag. Sci. 2020, 76, 868-879. [CrossRef] [PubMed]

20. Liu, X.H.; Yu, W.; Min, L.J.; Wedge, D.E.; Weng, J.Q.; Wu, H.-K.; Cantrell, C.L.; Bajsa-Hirschel, J.; Hua, X.-W. Synthesis and pesticidal activities of quinoxalines. J. Agric. Food Chem. 2020, 68, 7324-7332. [CrossRef]

21. Cheng, L.; Zhang, R.R.; Wu, H.K.; Xu, T.M.; Liu, X.H. The synthesis of 6-(tertbutyl)-8-fluoro-2,3-dimethylquinoline carbonate derivatives and their antifungal activity against Pyricularia oryzae. Front. Chem. Sci. Eng. 2019, 13, 369-376. [CrossRef]

22. Yao, T.T.; Xiao, D.X.; Li, Z.S.; Cheng, J.L.; Fang, S.W.; Du, Y.J.; Zhao, J.-H.; Dong, X.W.; Zhu, G.N. Design, synthesis, and fungicidal evaluation of novel pyrazole-furan and pyrazole-pyrrole carboxamide as succinate dehydrogenase inhibitors. J. Agric. Food Chem. 2017, 65, 5397-5403. [CrossRef]

23. Yao, T.T.; Fang, S.W.; Li, Z.S.; Xiao, D.X.; Cheng, J.L.; Ying, H.Z.; Du, Y.J.; Zhao, J.H.; Dong, X.W. Discovery of Novel Succinate Dehydrogenase Inhibitors by the Integration of in Silico Library Design and Pharmacophore Mapping. J. Agric. Food Chem. 2017, 65, 3204-3211. [CrossRef] [PubMed]

24. Wang, B.L.; Wang, H.X.; Liu, H.; Xiong, L.; Yang, N.; Zhang, Y.; Li, Z.M. Synthesis and structure-insectidical relationship of novel phenylpyrazole carboxylic acid dervatives containing fluorine moiety. Chin. Chem. Lett. 2020, 31, 739-745. [CrossRef]

25. Xue, H.S.; Liu, A.P.; Liu, W.D.; Li, J.M.; Ren, Y.G.; Huang, L.; He, L.; Ou, X.M.; Ye, J.; Huang, M.Z. Syntheses and fungicidal activities of thiazole-5-carboxanilides bearing thioether group. Chem. Res. Chin. Univ. 2016, 32, 781-785. [CrossRef]

26. King, W.F.; Wheeler, R.E. Substituted Oxadiazoles and Their Use as Corn Root Worm Insecticides. US Patent US4237121A, 2 December 1981. 
27. Liu, Q.; Zhu, R.; Gao, S.; Ma, S.H.; Tang, H.J.; Diao, Y.M.; Wang, H.L.; Zhu, H.J. Structure-based bioisosterism design, synthesis, insecticidal activity and structure-activity relationship (SAR) of anthranilic diamide analogues containing 1,2,4-oxadiazole rings. Pest Manage. Sci. 2017, 73, 917-924. [CrossRef]

28. Haugwitz, R.D.; Martinez, A.J.; Venslavsky, J.; Angel, R.G.; Maurer, B.V.; Jacobs, G.A.; Narayanan, V.L.; Cruthers, L.R.; Szanto, J. Synyhesis and anthelmintic acyivities of novel isothiocyanatophenyl-1,2,4-oxadiazoles. J. Med. Chem. 1985, 28, 1234-1241. [CrossRef]

29. Terteryan-Seiser, V.; Grammenos, W.; Wiebe, C.; Kretschmer, M.; Craig, I.R.; Escribano, C.A.; Marcus, F.; Tobias, M.; Palomar, M.A.Q.; Grote, T.; et al. Substituted Oxadiazoles for Combating Phytopathogenic Fungi. WO Patent WO2017178245A1, 19 October 2017.

30. Iwata, J.; Nakamura, Y.; Hayashi, T.; Watanabe, S.; Sano, H. Oxadiazole Compound and Fungicide for Agricultural and Horticultural Use. WO Patent WO2019022061A1, 31 January 2019.

31. Ryu, E.K.; Chung, K.H.; Lee, W.H.; Kim, J.N.; Hong, K.S. Herbicidal Quinolinyloxadiazoles. WO Patent WO9404530A1, 3 March 1994.

32. Feng, M.L.; Li, Y.F.; Zhu, H.J.; Zhao, L.; Xi, B.B.; Ni, J.P. Synthesis, Insecticidal Activity, and Structure-Activity Relationship of Trifluoromethyl-Containing Phthalic Acid Diamide Structures. J. Agric. Food Chem. 2010, 58, 10999-11006. [CrossRef] [PubMed]

33. Liu, M.; Wang, Y.; Wangyang, W.Z.; Liu, F.; Cui, Y.L.; Duan, Y.S.; Wang, M.; Liu, S.Z.; Rui, C.H. Design, Synthesis, and Insecticidal Activities of Phthalamides Containing a Hydrazone Substructure. J. Agric. Food Chem. 2010, 58, 6858-6863. [CrossRef]

34. Lahm, G.P.; Stevenson, T.M.; Selby, T.P.; Freudenberger, J.H.; Cordova, D.; Flexner, L.; Bellin, C.A.; Dubas, C.M.; Smith, B.K.; Hughes, K.A.; et al. Rynaxypyr ${ }^{\mathrm{TM}}$ : A new insecticidal anthranilic diamide that acts as a potent and selective ryanodine receptor activator. Bioorg. Med. Chem. Lett. 2007, 17, 6274-6279. [CrossRef]

35. Hughes, K.A.; Lahm, G.P.; Selby, T.P.; Stevenson, T.M. Cyano Anthranilamide Insecticides. WO Patent WO2004067528A1, 21 January 2004.

36. Clark, D.A.; Lahm, G.P.; Smith, B.K.; Barry, J.D.; Clagg, D.G. Synthesis of insecticidal fluorinated anthranilic diamides. Bioorg. Med. Chem. 2008, 16, 3163-3170. [CrossRef] [PubMed]

37. Wu, J.; Song, B.A.; Hu, D.Y.; Yue, M.; Yang, S. Design, synthesis and insecticidal activities of novel pyrazole amides containing hydrazone substructures. Pest Manag. Sci. 2012, 68, 801-810. [CrossRef] [PubMed]

38. Zhang, J.F.; Xu, J.Y.; Wang, B.L.; Li, Y.X.; Xiong, L.X.; Li, Y.Q.; Ma, Y.; Li, Z.M. Synthesis and insecticidal activities of novel anthranilic diamides containing acylthiourea and acylurea. J. Agric. Food Chem. 2012, 60, 7565-7572. [CrossRef]

39. Chen, K.; Liu, Q.; Ni, J.P.; Zhu, H.J.; Li, Y.F.; Wang, Q. Synthesis: Insecticidal activities and structureactivity relationship studies of novel anthranilic diamides containing pyridylpyrazole-4-carboxamide. Pest Manag. Sci. 2015, 71, 1503-1512. [CrossRef] [PubMed]

40. Hua, X.W.; Mao, W.T.; Fan, Z.J.; Ji, X.T. Novel anthranilic diamide insecticides: Design, synthesis, and insecticidal evaluation. Aust. J. Chem. 2014, 67, 1491-1503. [CrossRef]

41. Zhou, S.; Jia, Z.H.; Xiong, L.X.; Yan, T.; Yang, N.; Wu, G.; Song, H.; Li, Z. Chiral dicarboxamide scaffolds containing a sulfiliminyl moiety as potential ryanodine receptor activators. J. Agric. Food Chem. 2014, 62, 6269-6277. [CrossRef]

42. Zhou, S.; Gu, Y.C.; Liu, M.; Wu, C.; Zhou, S.; Zhao, Y.; Jia, Z.; Wang, B.; Xiong, L.; Yang, N.; et al. Insecticidal activities of chiral N-trifluoroacetyl sulfilimines as potential ryanodine receptor modulators. J. Agric. Food Chem. 2014, 62, 11054-11061. [CrossRef] [PubMed]

43. Amarasekare, K.G.; Shearer, P.W. Comparing effects of insecticides on two green lacewings species Chrysoperla johnsoni and Chrysoperla carnea (Neuroptera: Chrysopidae). J. Econ. Entomol. 2013, 106, 1126-1133. [CrossRef] [PubMed]

44. Schmidt, R.A.; Beers, E.H. Impacts of orchard pesticides on Galendromus occidentalis: Lethal and sublethal effects. Crop Prot. 2013, 56, 16-24.

45. Kohara, Y.; Kubo, K.; Imamiya, E.; Wada, T.; Inada, Y.; Naka, T. Synthesis and angiotensin II receptor antagonistic activities of benzimidazole derivatives bearing acidic heterocycles as novel tetrazole bioisosteres. J. Med. Chem. 1996, 39, 5228-5235. [CrossRef]

46. Tagad, H.D.; Hamada, Y.; Nguyen, J.T.; Hamada, T.; Abdel-Rahman, H.; Yamani, A.; Nagamine, A.; Ikari, H.; Igawa, N.; Hidaka, K.; et al. Design of pentapeptidic BACE1 inhibitors with carboxylic acid bioisosteres at P1' and P4 positions. Bioorg. Med. Chem. 2010, 18, 3175-3186. [CrossRef] [PubMed]

47. Yang, S.; Tian, X.Y.; Liu, X.H.; Tan, C.X. Synthesis and Biological Activity of Benzamides Substituted with Pyridine-Linked 1,2,4-Oxadiazole. Molecules 2020. [CrossRef] [PubMed]

48. Dey, S.; Mal, D. Total synthesis of BE-23254, a chlorinated angucycline antibiotic. Tetrahedron Lett. 2005, 46, 5483-5486. [CrossRef]

49. Zhang, Y.; Zhu, H.W.; Shang, J.F.; Wang, B.L.; Li, Z.M. Synthesis and Biological Activities of Novel 3-(((3-Bromo1-(3-chloropyridin2-yl)-1H-pyrazol-5-yl) methylene) amino)-substituted-benzo [d] [1-3] triazin-4(3H)-ones. Chin. J. Org. Chem. 2019, 39, 861-866. [CrossRef]

50. Mu, J.X.; Shi, Y.X.; Yang, M.Y.; Sun, Z.H.; Liu, X.H.; Li, B.J.; Sun, N.B. Design, Synthesis, DFT Study and Antifungal Activity of Pyrazolecarboxamide Derivatives. Molecules 2016, 21, 68. [CrossRef]

51. Zhang, Y.; Wang, X.; Yin, X.; Wang, H. Toxicity assessment of combined fluoroquinolone and tetracycline exposure in zebrafish (Danio rerio). Environ. Toxicol. Pharmacol. 2016, 31, 736-750. [CrossRef] [PubMed]

52. Zhang, Y.H.; Liu, M.; Liu, J.F.; Wang, X.; Wang, C.; Ai, W.; Chen, S.; Wang, H. Combined toxicity of triclosan 1,2,4-dichlorophenol and 2,4,6-trichlorophenol to zebrafish (Danio rerio). Environ. Toxicol. Pharmacol. 2018, 57, 9-18. [CrossRef] [PubMed] 